\title{
Tollmien-Schlichting wave cancellation via localised heating elements in boundary layers
}

DOI:

10.1017/jfm.2020.928

\section{Document Version}

Accepted author manuscript

Link to publication record in Manchester Research Explorer

\section{Citation for published version (APA):}

Brennan, G., Gajjar, J., \& Hewitt, R. (2020). Tollmien-Schlichting wave cancellation via localised heating elements in boundary layers. Journal of Fluid Mechanics, 909, [A16]. https://doi.org/10.1017/jfm.2020.928

\section{Published in:}

Journal of Fluid Mechanics

\section{Citing this paper}

Please note that where the full-text provided on Manchester Research Explorer is the Author Accepted Manuscript or Proof version this may differ from the final Published version. If citing, it is advised that you check and use the publisher's definitive version.

\section{General rights}

Copyright and moral rights for the publications made accessible in the Research Explorer are retained by the authors and/or other copyright owners and it is a condition of accessing publications that users recognise and abide by the legal requirements associated with these rights.

\section{Takedown policy}

If you believe that this document breaches copyright please refer to the University of Manchester's Takedown Procedures [http://man.ac.uk/04Y6Bo] or contact uml.scholarlycommunications@manchester.ac.uk providing relevant details, so we can investigate your claim.

\section{OPEN ACCESS}




\title{
Tollmien-Schlichting wave cancellation via localised heating elements in boundary layers
}

\author{
G. Brennan $^{1 *}$ and J. S. B. Gajjar ${ }^{1} \dagger$ and R.E. Hewitt ${ }^{1}$ \\ ${ }^{1}$ Department of Mathematics, University of Manchester, Manchester, M13 9PL,UK \\ * (current address):Mathematical Institute, University of Oxford, Oxford, OX2 6GG, UK
}

(Received $\mathrm{xx}$; revised $\mathrm{xx}$; accepted $\mathrm{xx}$ )

Instability to Tollmien-Schlichting waves is one of the primary routes to transition to turbulence for two-dimensional boundary layers in quiet disturbance environments. Cancellation of Tollmien-Schlichting waves using surface heating was first demonstrated in the experiments of Liepmann et al. (1982) and Liepmann \& Nosenchuck (1982). Here we consider a similar theoretical formulation that includes the effects of localised (unsteady) wall heating/cooling. The resulting problem is closely related to that of Terent'ev $(1981,1984)$ on the generation of Tollmien-Schlichting waves by a vibrating ribbon, but with thermal effects. The nonlinear receptivity problem based on tripledeck scales is formulated and the linearised version solved both analytically as well as numerically. The most significant result is that the wall heating/cooling function can be chosen such that there is no pressure response to the disturbance, meaning there is no generation of Tollmien-Schlichting waves. Numerical calculations substantiate this with an approximation based on the exact analytical result. Previous numerical studies of the unsteady triple-deck equations have shown difficulties in capturing the convective wavepacket that develops in the initial-value problem and we show that these arise from the choice of timesteps as well as the range of the Fourier modes taken.

Key words: Authors should not enter keywords on the manuscript, as these must be chosen by the author during the online submission process and will then be added during the typesetting process (see http://journals.cambridge.org/data/relatedlink/jfmkeywords.pdf for the full list)

\section{Introduction}

The classic experiments of Schubauer \& Skramstad (1948) demonstrated convincingly for the first time that small disturbances in the boundary layer could excite TollmienSchlichting instability and cause transition to turbulence. One of the important factors in their experiments was the use of a quiet wind tunnel together with controlled disturbances introduced with a vibrating ribbon on the flat plate. This led to unstable TollmienSchlichting waves which grow spatially downstream. Subsequent attempts to link the amplitude of the instability wave generated to the forcing disturbance amplitude, the so called receptivity problem, encountered many difficulties and objections as noted by Gaster (1965). The idea that a spatially growing disturbance wave could be described by theories of temporally growing unstable waves was not something that was readily

$\dagger$ Email address for correspondence: jitesh.gajjar@manchester.ac.uk 
accepted at that time. It wasn't until the advent of triple-deck theory that it was possble to provide a firm and rational mathematical foundation for describing the asymptotic properties of the instability. In fact triple-deck theory was first introduced by Stewartson \& Williams (1969), Neiland (1969) and Messiter (1970), in connection with self-induced separation in supersonic free interactions and to describe the boundary layer in the vicinity of the trailing-edge of a flat plate. It is now well known that for discussing the properties of flows with adverse pressure gradients and laminar separation, the classical Prandtl (1904) boundary layer theory does not work. The only self-consistent mathematical approach is based on studying interactions with the triple-deck scales, see for instance the extensive reviews of triple-deck theory by Stewartson $(1974,1981)$, and Smith (1982). The important connection between triple-deck theory and TollmienSchlichting instability was first enunciated by Smith (1979) who showed how the tripledeck scaling could be used to capture the behaviour of the lower-branch of the neutral curve predicted by Lin (1955) and others.

The work of Terent'ev $(1981,1984)$ provided an important and useful mathematical model for the vibrating ribbon experiments of Schubauer \& Skramstad (1948) based on triple-deck theory. In Terent'ev (1981) it was assumed that disturbances were generated by a vibrator oscillating harmonically in time in the wall normal direction. In the Terent'ev (1984) paper, the problem was modified to study the initial-value problem instead of periodic motion. The Terent'ev (1984) work showed how unstable, spatially growing instability waves could be triggered by the wall motion and the critical frequencies were in agreement with the results of Smith (1979). More importantly the Terent'ev (1981, 1984) results showed how the amplitude of the resulting downstream travelling instability wave could be calculated based on the amplitude of the forcing disturbance.

The receptivity problem studied by Terent'ev $(1981,1984)$ is of course somewhat special in that Tollmien-Schlicting instability waves may be triggered by various means including free-stream turbulence, the shape of the leading edge, wall roughness and various other factors, and not just controlled wall motion such as that induced by a vibrator. Ruban (1984) and Goldstein $(1983,1985)$ showed how the receptivity coefficients could be calculated for waves induced by acoustic noise and leading edges. The paper by De Tullio \& Ruban (2015) summarizes more recent progress in this area and highlights the value of the asymptotic approach in problems of this type.

The use of surface heating/cooling as a means for flow control has been of considerable interest especially in many aerospace related applications. It was shown by Liepmann et al. (1982) and Liepmann \& Nosenchuck (1982) that surface heating could be used to excite Tollmien-Schlicting waves in a similar manner to using a vibrating ribbon. More importantly these papers were one of the earliest to demonstrate the cancellation of Tollmien-Schlicting waves by using an array of surface heating strips. In their experiments one heating strip was used to generate an unstable wave and another heating strip positioned further downstream, could be used to either reinforce or cancel the generated wave via a feedback loop.

The comprehensive review by Löfdahl \& Gad-el-Hak (1999) discusses the use of microelectromechanical-systems (MEMS) type devices for controlling many different types of turbulent flows. MEMS devices have characteristic lengths between $1 \mu \mathrm{m}$ to $1 \mathrm{~mm}$, commensurate with boundary layer scales, and they have the requisite spatial and temporal response characteristics suitable for use in active flow control of boundary layer type instabilities. Lipatov (2006) was one of the first to try and develop a mathematical model based on triple-deck theory to understand how localised heating elements affect flow properties. He suggested that localised heating creates thermal humps akin to physical humps which lead to an interaction with the oncoming flow. For modeling MEMS 
type devices, the choice of triple-deck scales, as opposed to other scales is discussed in detail in Lipatov (2006) and Koroteev \& Lipatov (2009).

The ideas suggested by Lipatov (2006) have been applied to a variety of other situations involving predominantly steady localised heating including subsonic and supersonic flows, see Koroteev \& Lipatov $(2009,2012,2013)$. In a series of recent papers Aljohani \& Gajjar $(2017 a, b, 2018)$ have investigated steady boundary layer flow with localised heating but over hump-shaped elements to understand how localised heating can affect a separated flow. Both two-dimensional as well as three-dimensional hump-shaped elements were studied for an oncoming subsonic or transonic flow. It was found that localised heating can have beneficial properties leading to more attached flow over the hump, although near the forward and rear parts of the hump the wall shear has more pronounced minimum values. These findings are not too dissimilar to earlier work by Koroteev \& Lipatov (2012) who studied localised heating over flat-plate elements.

One of the objectives of the current work is to investigate the stability of the flow considered by Aljohani \& Gajjar (2017a). The work in that paper is based on tripledeck scales and so the most natural starting point is to look at unsteady effects which appear non-trivially in the lower-deck. The governing equations in this case reduce to the modified unsteady triple-deck equations with an additional unsteady equation for the perturbation temperature. The problem we study here is in fact the linear receptivity of a boundary layer flow to a vibrator on the wall together with localised heating effects This is a mathematical model of the experiments of Liepmann et al. (1982), Liepmann \& Nosenchuck (1982) and is a generalisation of the vibrating ribbon problem first studied by Terent'ev $(1981,1984)$.

We first formulate the initial-value problem, which is solved both analytically and numerically. The most significant result of our work, and one which has not been identified previously, is that an appropriate choice of unsteady temperature distribution exists that will cancel the Tollmien-Schlichting wave that would otherwise be generated from the wall vibrator. An exact formula is provided for the required temperature distribution together with a simplified approximation to the cancellation function. Numerical results confirm that significant reduction in Tollmien-Schlicting wave amplitudes can be achieved by the simplified expression. A stabilisation of lower-branch instability waves in the free convection flow over a heated element was also identified in the paper by Trevin̄o \& Lin̄án (1996), whilst in Seddougui et al. (1991) wall cooling is used to destabilize viscous and inviscid modes in the boundary layer.

Previous studies involving numerical simulations of the unsteady triple-deck equations have encountered difficulties and unexplained behaviour in the results. For example in the recent paper by Logue et al. (2014) on unsteady flow past a compression ramp the results showed the development of a wave packet which grew in amplitude and convected downstream. The techniques used in Logue et al. (2014) to solve the unsteady triple-deck equations utilized high-order finite differencing in the streamwise direction, combined with Chebychev collocation in the wall-normal direction, and a variety of time-marching schemes were tested. In grid refinement studies, the wave packet signal was not resolved spatially or temporally as seen in figure 6 of Logue et al. (2014). No convincing explanation was available to explain this behaviour. The same behaviour was also observed in related unsteady simulations of the triple-deck equations for jet and liquid layer flows and subsonic flows, see Logue (2008), using similar numerical techniques. In other independent simulations of unsteady compression ramp flow using very different numerical techniques, Cassel et al. (1995) and Fletcher et al. (2004), observed analagous wave packet behaviour. Cassel et al. (1995) suggested that this was linked to the Tutty \& Cowley (1986) short wavelength inflexion point instability. However, this explanation is 
unconvincing as the underlying base flow does not contain inflexion points in the range of ramp angle parameters when the phenomenon is first observed. Likewise Fletcher et al. (2004) suggest absolute instabilities of the base flow. Such explanations are dismissed in the more careful investigations by Logue et al. (2014) where it is noted that none of the claims have any supporting underlying evidence.

Another motivation for the current work is therefore to examine a much simplified problem involving just the linearised unsteady triple-deck equations, with the base flow being the linear shear profile. By doing this we can study the evolution of the perturbations using a similar numerical technique to that of Logue et al. (2014) to see if anything new can be learnt about the wave packets that arise in the simulations, and whether the unexplained difficulties can be resolved. The Terent'ev (1984) vibrator problem, or the generalisation of it with localised heating as adopted here, is one such simpler problem where it is also possible to obtain a solution using analytical means. Here we use a Fourier-Laplace transform to solve the initial-value problem and obtain the analytical results in addition to solving the same equations numerically. The results presented in the current work demonstrate that a wave packet, emanating from the forced response, grows to large amplitudes very quickly, as indeed also seen in the experiments of Gaster \& Grant (1975). The numerical difficulties in resolving the wave packet occur because of the timesteps used in the numerical solution were too large, as well as from the number of Fourier modes taken. By reducing the timesteps used and working with a restricted range of wave numbers in Fourier space, the signal can be resolved both spatially and temporally.

In section 2 below we derive the governing unsteady equations starting from the compressible Navier-Stokes equations and using the scalings appropriate to the lowerbranch. The full motivation for using the chosen scalings and the inclusion of localised heating effects is given in other papers, see Koroteev \& Lipatov (2009), Aljohani \& Gajjar $(2017 b)$ for instance, and not repeated here. The adoption of the scalings appropriate to the lower-branch instability of the boundary layer is linked to the objectives discussed above. The problem formulation for flow over localised heating with upper-branch scalings can be obtained as a special case of the problem formulation by Gajjar (1996). In section 3 we discuss the analytical solution to the linearised unsteady equations using Fourier-Laplace transforms. In section 4 a numerical solution of the linearised unsteady equations, is obtained via a time-stepping algorithm combining spectral collocation in the wall normal direction and solving for the individual wavenumbers in Fourier space. The numerical techniques used are very similar to those of Logue et al. (2014). Finally in section 5 we finish with some additional comments and conclusions.

\section{Problem formulation}

The problem we study here is a modified version of one first studied by Terent'ev (1981). Consider the subsonic flow past a flat plate containing a vibrator at a distance $L$ from the leading edge of the plate, see Fig. 1. The plate also contains a localised heating element whose dimensions are small compared with the thickness of the boundary layer. We will assume that the Reynolds number $R e$ is large (where $R e=\rho_{\infty} U_{\infty} L / \mu_{\infty}$ ). At large distances from the plate the flow is uniform with speed $U_{\infty}$ parallel to the plate and with density $\rho_{\infty}$ and $\mu_{\infty}$ is the dynamic viscosity coefficient. We will assume that the flow is two-dimensional and neglect any variations in the spanwise direction.

We non-dimensionalise the variables and flow quantities with respect to a lengthscale 

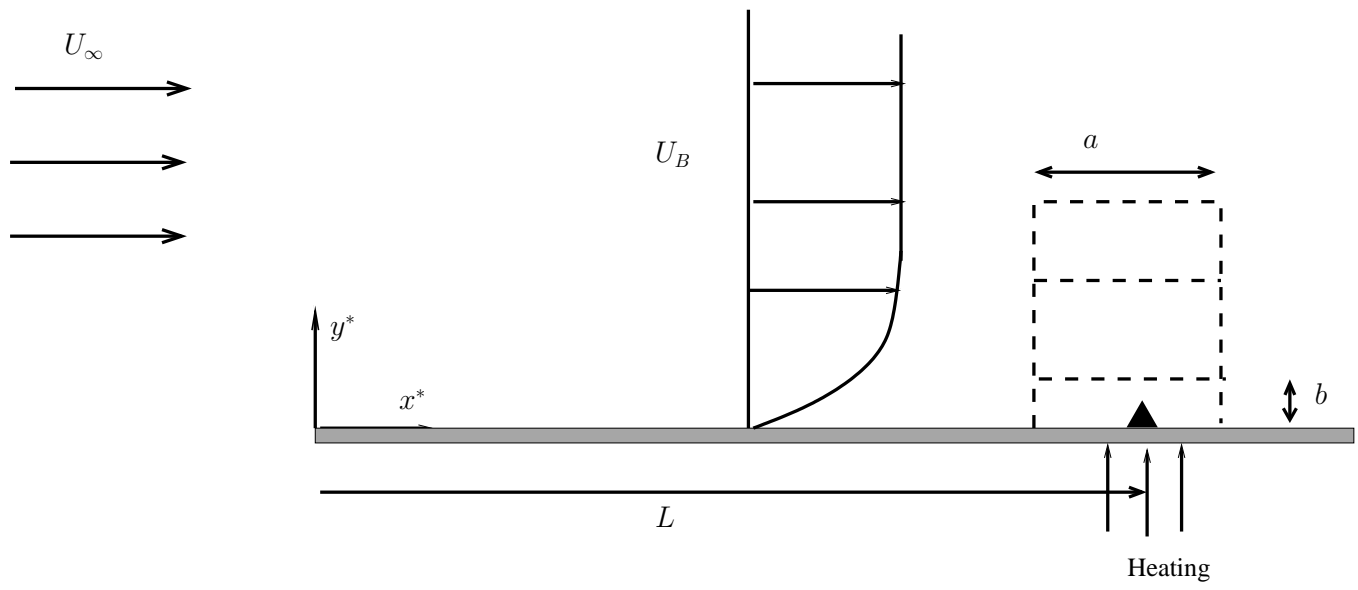

Figure 1: Boundary layer flow over a flat plate with speed $U_{\infty}$ at large distances from the plate. A vibrator is located at a distance $L$ from the leading edge and with an oncoming boundary layer flow represented by $U_{B}$.

$L$, velocity $U_{\infty}$, and freestream density $\rho_{\infty}$ so that

$$
\begin{gathered}
x=\frac{x^{*}}{L}, \quad y=\frac{y^{*}}{L}, \quad t=\frac{U_{\infty}}{L} t^{*}, \quad u=\frac{u^{*}}{U_{\infty}}, \quad v=\frac{v^{*}}{U_{\infty}}, \quad T=\frac{T^{*}}{T_{\infty}}, \\
p=\frac{p^{*}-p_{\infty}}{\rho_{\infty} U_{\infty}^{2}}, \quad \mu=\frac{\mu^{*}}{\mu_{\infty}} \quad \text { and } \quad \rho=\frac{\rho^{*}}{\rho_{\infty}} .
\end{gathered}
$$

The superscript asterisk quantities are dimensional, $(x, y)$ are the coordinates in the streamwise and wall normal direction with corresponding velocity components $(u, v), t$ is time, $T$ is the temperature, $p$ the pressure, $\mu$ the dynamic viscosity and $\rho$ is the density, $p_{\infty}$ is the free-stream pressure.

In addition to the Reynolds number $R e$ we have the Prandtl number $P r$, the specific gas constant $\mathcal{R}$, and $M_{\infty}$ the free-stream Mach number. Here, $M_{\infty}=U_{\infty} / c_{\infty}$ where $c_{\infty}=\sqrt{\gamma p_{\infty} / \rho_{\infty}}$ is the speed of sound in the undisturbed flow and $\gamma$ is the ratio of specific heats, and $T_{\infty}=U_{\infty}^{2} /\left(M_{\infty}^{2} \mathcal{R} \gamma\right)$.

We will assume that the vibrator oscillates with a frequency $\Omega$ and has a maximum amplitude $b$ in the transverse direction. The vibrator is confined to a distance $a$ in the streamwise direction. Whilst it is possible to consider different scenarios, we will restrict our attention to the case when $b=O\left(R e^{-5 / 8}\right)$, and $a=O\left(R e^{-3 / 8}\right)$. These are precisely the triple-deck scalings which, as shown by Smith (1979), capture the asymptotic properties of the dominant Tollmien-Schlichting wave instability in the boundary layer. With this choice of scales it is possible to make further progress using analytical techniques. Starting with the triple-deck structure also allows other distinguished limits to be studied as limiting cases of the current problem. Lipatov (2006) has suggested additional possibilities which arise from choosing different scalings for $a$ and $b$ when localised surface heating is present.

We need additionally to decide on the time scale to be used in the analysis. Choosing the time scale such that the unsteady terms appear at the same time as nonlinearity in the wall layer would appear to the most natural starting point. Other distinguished scales may then be deduced via limiting cases. Suppose $\Delta u$ denotes the perturbation to the oncoming flow, and $\Delta x=O\left(R e^{-3 / 8}\right)$ is the streamwise extent of the perturbation. 
If we balance the unsteady and inertial terms in the wall layer we find that

$$
\frac{\partial u}{\partial t} \sim u \frac{\partial u}{\partial x}
$$

gives

$$
\frac{\Delta u}{\Delta t} \sim u \frac{\Delta u}{\Delta x}
$$

where $\Delta t$ denotes the scale of the unsteady variations in the wall layer. The streamwise velocity perturbation in the lower-deck is $u \sim \Delta u=O\left(R e^{-\frac{1}{8}}\right)$.

Hence

$$
\Delta t \sim \frac{\Delta x}{u} \sim \frac{R e^{-\frac{3}{8}}}{R e^{-\frac{1}{8}}}=R e^{-\frac{1}{4}} .
$$

With the above scalings, the vibrator is situated near $x=1$ and hence we may set $x=1+R e^{-\frac{3}{8}} x_{*}$ where $x_{*}$ is $O(1)$ in the region occupied by the vibrator. We will model the vibrator by some function $y=y_{w}(t, x)$ with

$$
y=y_{w}(t, x)=R e^{-\frac{5}{8}} f\left(t_{*}, x_{*}\right),
$$

and the function $f\left(t_{*}, x_{*}\right)$ describes the spatial distribution of the localised disturbance caused by the vibrator. Specific forms of $f\left(t_{*}, x_{*}\right)$ will be considered later, but it is assumed that $f\left(t_{*}, x_{*}\right)$ is zero apart from a small interval in the vicinity of the vibrator.

The presence of the vibrator means that at the wall we will require that

$$
u=0, \quad v=\frac{\partial y_{w}}{\partial t} \quad \text { on } \quad y=y_{w}(t, x)
$$

In view of the earlier comments regarding scalings we will set the frequency of oscillation $\Omega=\omega_{0}^{*} R e^{\frac{1}{4}}$, where $\omega_{0}^{*}$ will be taken to be an $O(1)$ scaled frequency parameter. Let $t=R e^{-\frac{1}{4}} t_{*}$ and $t_{*}$ is $O(1)$.

Next we assume that there is also a localised heating element on the flat plate colocated with the vibrator. This is modelled by the wall temperature profile being given by $T=T_{w}\left(t_{*}, x_{*}\right)$ on $y=y_{w}$.

Unsteady effects notwithstanding, the analysis leading to the reduced governing equations follows closely that of the steady problem of subsonic flow over a localised heated element as in Aljohani \& Gajjar (2017b) and so are not repeated here. The fundamental problem reduces to solving the triple-deck equations in the lower-deck, see figure 2, where (with $y=R e^{-5 / 8} y_{3}$ ) the flow quantities are expanded as:

$$
\begin{aligned}
& u(t, x, y ; R e)=R e^{-\frac{1}{8}} u_{*}\left(t_{*}, x_{*}, y_{3}\right)+\ldots, \\
& v(t, x, y ; R e)=R e^{-\frac{3}{8}} v_{3}\left(t_{*}, x_{*}, y_{3}\right)+\ldots, \\
& p(t, x, y ; R e)=R e^{-\frac{1}{4}} p_{*}\left(t_{*}, x_{*}, y_{3}\right)+\ldots, \\
& \rho(t, x, y ; R e)=\rho_{*}\left(t_{*}, x_{*}, y_{3}\right)+\ldots \\
& T(t, x, t ; R e)=T_{*}\left(t_{*}, x_{*}, y_{3}\right)+\ldots
\end{aligned}
$$

Substitution into the Navier-Stokes, the continuity equation, the energy equation and the equation of state, gives:

$$
\begin{aligned}
\frac{\partial \rho_{*}}{\partial t_{*}}+\frac{\partial\left(\rho_{*} u_{*}\right)}{\partial x_{*}}+\frac{\partial\left(\rho_{*} v_{3}\right)}{\partial y_{3}} & =0 \\
\rho_{*}\left(\frac{\partial u_{*}}{\partial t_{*}}+u_{*} \frac{\partial u_{*}}{\partial x_{*}}+v_{3} \frac{\partial u_{*}}{\partial y_{3}}\right) & =-\frac{\partial p_{*}}{\partial x_{*}}+\frac{\partial}{\partial y_{3}}\left(\mu_{*} \frac{\partial u_{*}}{\partial y_{3}}\right),
\end{aligned}
$$




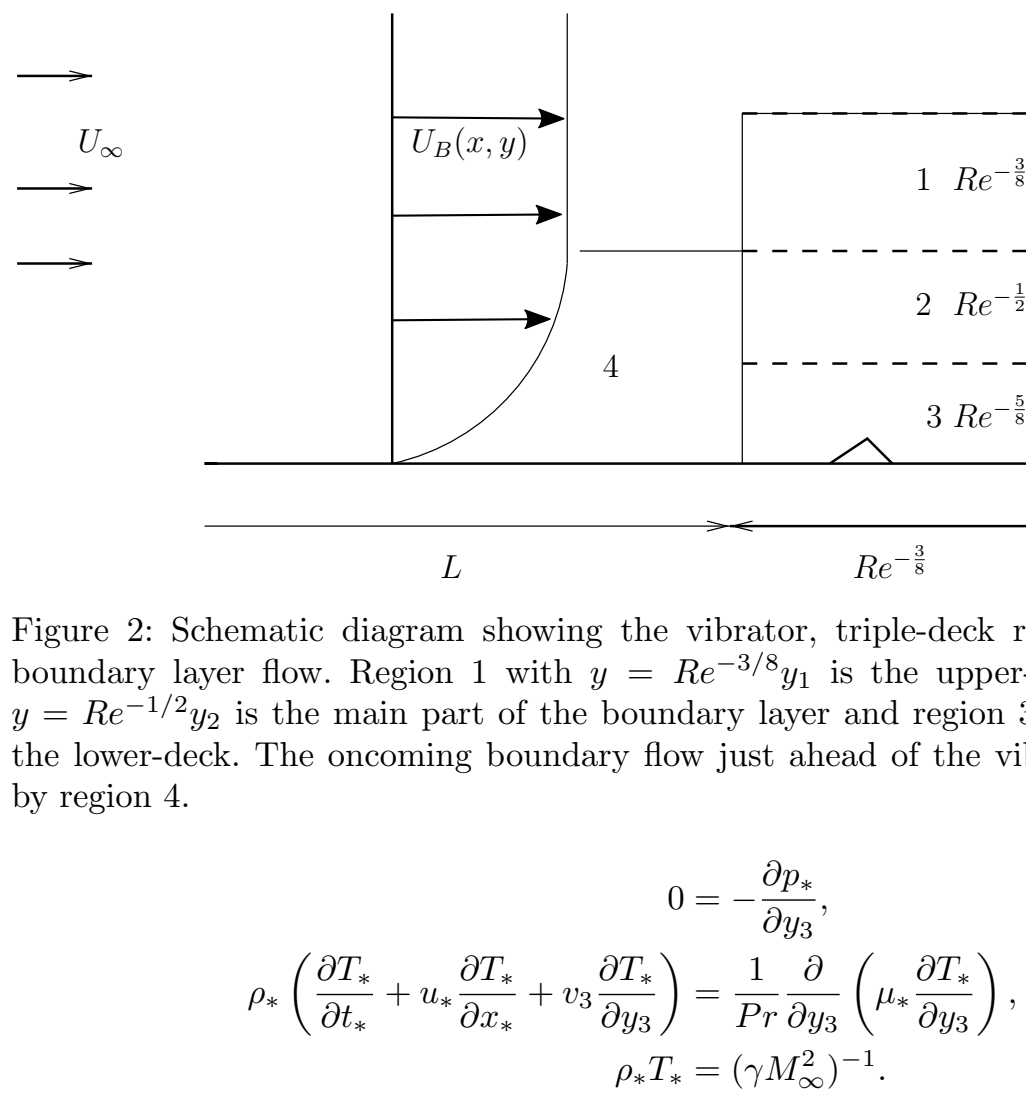

The boundary conditions are:

$$
\begin{aligned}
v_{3}\left(t_{*}, x_{*}, y_{3}\right) & =\frac{\partial f}{\partial t_{*}} & & \text { on } \quad y_{3}=f\left(t_{*}, x_{*}\right), \\
u_{*}\left(t_{*}, x_{*}, y_{3}\right) & =0 & & \text { on } \quad y_{3}=f\left(t_{*}, x_{*}\right), \\
T_{*}\left(t_{*}, x_{*}, y_{3}\right) & =T_{w}\left(t_{*}, x_{*}\right) & & \text { on } \quad y_{3}=f\left(t_{*}, x_{*}\right), \\
u_{*}\left(t_{*}, x_{*}, y_{3}\right) & =\lambda y_{3} & & \text { as } \quad x_{*} \rightarrow-\infty \\
A_{*}\left(t_{*}, x_{*}\right) & =0 & & \text { as } \quad x_{*} \rightarrow-\infty \\
T_{*}\left(t_{*}, x_{*}, y_{*}\right) & =\left(\gamma M_{\infty}^{2}\right)^{-1} & & \text { as } \quad x_{*} \rightarrow-\infty \\
u_{*}\left(t_{*}, x_{*}, y_{3}\right) & =\lambda\left(y_{3}+A_{*}\left(t_{*}, x_{*}\right)\right) & & \text { as } \quad y_{3} \rightarrow \infty \\
T_{*}\left(t_{*}, x_{*}, y_{*}\right) & =\left(\gamma M_{\infty}^{2}\right)^{-1} & & \text { as } \quad y_{3} \rightarrow \infty
\end{aligned}
$$

with $\lambda=\left.\frac{\partial U_{B}(1, y)}{\partial y}\right|_{y=0}$ being the reduced shear of the oncoming boundary layer flow $u=$ $U_{B}\left(x_{*}, R e^{1 / 2} y\right)$, and $A_{*}$ is an unknown displacement function. Notice that even though the boundary conditions $(2.4 f),(2.4 h)$ suggest that the temperature is constant when merging with oncoming flow, the temperature does vary in the lower-deck interaction region as governed by $(2.3 d)$ and is also not constant in region 4 where there is a thermal boundary layer.

The above equations are coupled with the following upper-deck (region 1) problem which is the same as in the steady case, see for example Stewartson (1974):

$$
\left(1-M_{\infty}^{2}\right) \frac{\partial^{2} p_{1}}{\partial x_{*}^{2}}+\frac{\partial^{2} p_{1}}{\partial y_{1}^{2}}=0,
$$


with $y=R e^{-3 / 8} y_{1}$ and the pressure has been expanded as

$$
p=R e^{-\frac{1}{4}} p_{1}\left(t_{*}, x_{*}, y_{1}\right)+\ldots
$$

The problem for $p_{1}$ is to be solved together with the matching conditions

$$
p_{1}\left(t_{*}, x_{*}, y_{1}=0\right)=p_{*}\left(t_{*}, x_{*}\right),\left.\quad \frac{\partial p_{1}}{\partial y_{1}}\right|_{y_{1}=0}=\frac{\partial^{2} A_{*}\left(t_{*}, x_{*}\right)}{\partial x_{*}^{2}},
$$

and far field conditions

$$
p_{*}\left(t_{*}, x_{*}, y_{1}\right) \rightarrow 0 \quad \text { as } \quad x_{*}^{2}+y_{1}^{2} \rightarrow \infty .
$$

We first make use of the combined unsteady Dorodnitsyn-Howarth transform followed by the Prandtl transposition given by

$$
t_{*}, x_{*}, y_{*} \rightarrow t_{*}, x_{*}, \quad y_{*}\left(t_{*}, x_{*}, y_{3}\right)=\int_{f\left(t_{*}, x_{*}\right)}^{y_{3}} \rho\left(t_{*}, x_{*}, y_{3}\right) d y_{3},
$$

and

$$
\rho_{*} v_{3}=v_{*}-\frac{\partial y_{*}}{\partial t_{*}}-u_{*} \frac{\partial y_{*}}{\partial x_{*}} .
$$

The form of the transform above with the lower limit non-zero is an extension of the usual unsteady Dorodnitsyn-Howarth transform which is discussed in van Dyke (1952) for the one-dimensional case, and Neiland et al. (2007) for the two-dimensional case. The equations (2.3) and boundary conditions (2.4) reduce (see Appendix A for details) to:

$$
\begin{aligned}
\frac{\partial u_{*}}{\partial x_{*}}+\frac{\partial v_{*}}{\partial y_{*}} & =0 \\
\frac{\partial u_{*}}{\partial t_{*}}+u_{*} \frac{\partial u_{*}}{\partial x_{*}}+v_{*} \frac{\partial u_{*}}{\partial y_{*}} & =-T_{*}\left(\gamma M_{\infty}^{2}\right) \frac{\partial p_{*}}{\partial x_{*}}+\frac{\partial}{\partial y_{*}}\left(\rho_{*} \mu_{*} \frac{\partial u_{*}}{\partial y_{*}}\right), \\
0 & =-\frac{\partial p_{*}}{\partial y_{*}}, \\
\frac{\partial T_{*}}{\partial t_{*}}+u_{*} \frac{\partial T_{*}}{\partial x_{*}}+v_{*} \frac{\partial T_{*}}{\partial y_{*}} & =\frac{1}{\operatorname{Pr}} \frac{\partial}{\partial y_{*}}\left(\rho_{*} \mu_{*} \frac{\partial T_{*}}{\partial y_{*}}\right), \\
\rho_{*} T_{*} & =\left(\gamma M_{\infty}^{2}\right)^{-1} .
\end{aligned}
$$

The boundary conditions are:

$$
\begin{array}{ll}
v_{*}\left(t_{*}, x_{*}, y_{*}\right)=0 & \text { on } y_{*}=0, \\
u_{*}\left(t_{*}, x_{*}, y_{*}\right)=0 & \text { on } y_{*}=0, \\
T_{*}\left(t_{*}, x_{*}, y_{*}\right)=T_{w}\left(t_{*}, x_{*}\right) & \text { on } y_{*}=0, \\
u_{*}\left(t_{*}, x_{*}, y_{*}\right)=\lambda y_{*} & \text { as } \quad x_{*} \rightarrow-\infty, \\
u_{*}\left(t_{*}, x_{*}, y_{*}\right)=\lambda\left(y_{*}+K_{*}\left(t_{*}, x_{*}\right)\right) & \text { as } \quad y_{*} \rightarrow \infty,
\end{array}
$$

where

$$
K_{*}\left(t_{*}, x_{*}\right)=f\left(t_{*}, x_{*}\right)+\int_{0}^{\infty}\left(\gamma M_{\infty}^{2} T_{*}-1\right) d y_{*}+A_{*}\left(t_{*}, x_{*}\right) .
$$

The term involving the integral in the expression for $K_{*}$ in $(2.7 f)$ represents the additional displacement effect produced by the wall heating.

In what follows we use the Chapman viscosity law expressed by $\mu_{*}=C T_{*}$ for some constant $C$. This and additional constants such as $\lambda, \gamma, M_{\infty}$ appearing in the above 
equations may be effectively removed with the aid of the following affine transformation:

$$
\begin{gathered}
t_{*}=\beta^{1 / 2} \lambda^{-3 / 2} C^{-1 / 2}\left(\gamma M_{\infty}^{2}\right)^{1 / 2} \tau, \quad x_{*}=\beta^{3 / 4} \lambda^{-5 / 4}\left(\gamma M_{\infty}^{2}\right)^{1 / 4} C^{-1 / 4} X, \\
y_{*}=\lambda^{-3 / 4} \beta^{1 / 4} C^{1 / 4}\left(\gamma M_{\infty}^{2}\right)^{-1 / 4} Y, \quad y_{1}=\lambda^{-5 / 4} \beta^{7 / 4} C^{-1 / 4}\left(\gamma M_{\infty}^{2}\right)^{1 / 4} \bar{Y}, \\
u_{*}=\lambda^{\frac{1}{4}} \beta^{1 / 4} C^{1 / 4}\left(\gamma M_{\infty}^{2}\right)^{-1 / 4} U, \quad v_{*}=\lambda^{3 / 4} C^{3 / 4} \beta^{-1 / 4}\left(\gamma M_{\infty}^{2}\right)^{-3 / 4} V, \\
p_{*}=\beta^{1 / 2} \lambda^{1 / 2} C^{1 / 2}\left(\gamma M_{\infty}^{2}\right)^{-1 / 2} P, \quad T_{*}=\left(\gamma M_{\infty}^{2}\right)^{-1} \theta, \quad p_{1}=\beta^{1 / 2} \lambda^{1 / 2} C^{1 / 2}\left(\gamma M_{\infty}^{2}\right)^{-1 / 2} P_{1}, \\
A_{*}=\lambda^{-3 / 4} \beta^{1 / 4} C^{1 / 4}\left(\gamma M_{\infty}^{2}\right)^{-1 / 4} A, \quad K_{*}=\lambda^{-3 / 4} \beta^{1 / 4} C^{1 / 4}\left(\gamma M_{\infty}^{2}\right)^{-1 / 4} K, \\
f=\lambda^{-3 / 4} \beta^{1 / 4} C^{1 / 4}\left(\gamma M_{\infty}^{2}\right)^{-1 / 4} F, \quad T_{w}=\left(\gamma M_{\infty}^{2}\right)^{-1} \theta_{w},
\end{gathered}
$$

and we have put $\beta=\left(1-M_{\infty}^{2}\right)^{-1 / 2}$. After using the above transformation in (2.6)-(2.7) in conjunction with the Chapman viscosity law and the equation of state the resulting equations are given by:

$$
\begin{aligned}
& \frac{\partial U}{\partial X}+\frac{\partial V}{\partial Y}=0 \\
& \frac{\partial U}{\partial \tau}+U \frac{\partial U}{\partial X}+V \frac{\partial U}{\partial Y}=-\theta \frac{\partial P}{\partial X}+\frac{\partial^{2} U}{\partial Y^{2}}, \\
& 0=-\frac{\partial P}{\partial Y} \\
& \frac{\partial \theta}{\partial \tau}+U \frac{\partial \theta}{\partial X}+V \frac{\partial \theta}{\partial Y}=\frac{1}{\operatorname{Pr}} \frac{\partial^{2} \theta}{\partial Y^{2}} . \\
& V(\tau, X, Y)=0 \quad \text { on } Y=0, \\
& U(\tau, X, Y)=0 \quad \text { on } Y=0 \text {, } \\
& \theta(\tau, X, Y)=\theta_{w}(\tau, X) \quad \text { on } \quad Y=0 \text {, } \\
& U(\tau, X, Y)=Y \quad \text { as } X \rightarrow-\infty \text {, } \\
& U(\tau, X, Y)=Y+K(\tau, X), \quad \text { as } \quad Y \rightarrow \infty .
\end{aligned}
$$

Here $K$ is given by

$$
K(\tau, X)=F(\tau, X)+\int_{0}^{\infty}(\theta(\tau, X, Y)-1) d Y+A(\tau, X) .
$$

The transformed upper-deck problem is

$$
\frac{\partial^{2} P_{1}}{\partial X^{2}}+\frac{\partial^{2} P_{1}}{\partial \bar{Y}^{2}}=0
$$

with the boundary conditions

$$
\begin{aligned}
P_{1} & \rightarrow 0 \quad \text { as } \quad\left(X^{2}+\bar{Y}^{2}\right) \rightarrow \infty \\
P_{1}(\tau, X, \bar{Y}=0) & =P(\tau, X), \quad \frac{\partial P_{1}}{\partial \bar{Y}}=\frac{\partial^{2} A}{\partial X^{2}} \quad \text { on } \quad \bar{Y}=0 .
\end{aligned}
$$

In the above $F$ represents the transformed wall shape and $\theta_{w}$ is the prescribed heating profile and both these functions are assumed to be given.

The above initial value problem is supplemented with the initial conditions

$$
U=Y, \quad \theta=\theta_{w}=1, \quad V, P, P_{1}, A, F=0 \quad \text { for } \quad \tau \leqslant 0 .
$$

The nonlinear initial-value problem requires a numerical solution in general, but for small amplitudes of the vibrator we can find a linearised solution. 
We will assume that the wall motion and localised heating profiles are given by

$$
\begin{gathered}
F(\tau, X)=\epsilon F_{a}(\tau, X)=\epsilon h(X) \sin \left(\omega_{0} \tau\right), \quad \tau>0, \\
\theta_{w}(\tau, X)=1+\epsilon g(\tau, X), \quad \tau>0 .
\end{gathered}
$$

where $\epsilon$ represents the maximum amplitude of the oscillation and $\omega_{0}$ is some prescribed frequency. If $g=0$ the above reduces to the problem studied by Terent'ev (1984).

\subsection{Fourier-Laplace solution for small $\epsilon$}

For $0<\epsilon \ll 1$ we may expand the flow quantities as

$$
\begin{aligned}
U(\tau, X, Y) & =Y+\epsilon U_{a}(\tau, X, Y)+O\left(\epsilon^{2}\right), \\
V(\tau, X, Y) & =\epsilon V_{a}(\tau, X, Y)+O\left(\epsilon^{2}\right), \\
\theta(\tau, X, Y) & =1+\epsilon \theta_{a}(\tau, X, Y)+O\left(\epsilon^{2}\right), \\
P(\tau, X) & =\epsilon P_{a}(\tau, X)+O\left(\epsilon^{2}\right), \\
P_{1}(\tau, X, \bar{Y}) & =\epsilon P_{u}(\tau, X, \bar{Y})+O\left(\epsilon^{2}\right), \\
A(\tau, X) & =\epsilon A_{a}(\tau, X)+O\left(\epsilon^{2}\right), \\
K(\tau, X) & =\epsilon K_{a}(\tau, X)+O\left(\epsilon^{2}\right) .
\end{aligned}
$$

Substituting (2.12) into (2.8)-(2.10) and linearising for small $\epsilon$ leads to the following linearised initial-value problem

$$
\begin{aligned}
\frac{\partial U_{a}}{\partial X}+\frac{\partial V_{a}}{\partial Y} & =0 \\
\frac{\partial U_{a}}{\partial \tau}+Y \frac{\partial U_{a}}{\partial X}+V_{a} & =-\frac{\partial P_{a}}{\partial X}+\frac{\partial^{2} U_{a}}{\partial Y^{2}}, \\
0 & =-\frac{\partial P_{a}}{\partial Y}, \\
\frac{\partial \theta_{a}}{\partial \tau}+Y \frac{\partial \theta_{a}}{\partial X} & =\frac{1}{P r} \frac{\partial^{2} \theta_{a}}{\partial Y^{2}}, \\
K_{a} & =h(X) \sin \left(\omega_{0} \tau\right)+A_{a}+\int_{0}^{\infty} \theta_{a} d Y .
\end{aligned}
$$

where

$$
\begin{aligned}
U_{a}(\tau, X, Y=0) & =0, \\
V_{a}(\tau, X, Y=0) & =0, \\
\theta_{a}(\tau, X, Y=0) & =g(\tau, X), \\
U_{a}=V_{a}=\theta_{a}=K_{a}=P_{a}=A_{a} & =0 \text { for } \tau \leqslant 0, \\
U_{a}(\tau, X, Y) & =0 \text { as } X \rightarrow-\infty, \\
U_{a}(\tau, X, Y) & =K_{a}(\tau, X) \quad \text { as } \quad Y \rightarrow \infty,
\end{aligned}
$$

and

with the boundary conditions

$$
\frac{\partial^{2} P_{u}}{\partial X}+\frac{\partial^{2} P_{u}}{\partial \bar{Y}^{2}}=0
$$

$$
\begin{aligned}
P_{u} & \rightarrow 0 \quad \text { as } \quad\left(X^{2}+\bar{Y}^{2}\right) \rightarrow \infty \\
P_{u}(\tau, X, \bar{Y}=0) & =P_{a}(\tau, X), \quad \frac{\partial P_{u}}{\partial \bar{Y}}=\frac{\partial^{2} A_{a}}{\partial X^{2}} \quad \text { on } \quad \bar{Y}=0 .
\end{aligned}
$$


Let us introduce the Fourier-Laplace transform

$$
U_{a}^{\dagger \dagger}(\omega, k, Y)=\int_{0}^{\infty} \int_{-\infty}^{\infty} U_{a}(\tau, X, Y) e^{-\omega \tau-i k X} d X d \tau
$$

and the corresponding inverse by

$$
U_{a}(\tau, X, Y)=\frac{1}{4 \pi^{2} i} \int_{-\infty}^{\infty} \int_{\mathcal{L}} U_{a}^{\dagger \dagger}(\omega, k, Y) e^{\omega \tau+i k X} d \omega d k,
$$

with similar expressions for the other quantities. The double superscript denotes the Fourier-Laplace transform and the single superscript the Fourier transform. Also $\mathcal{L}$ is a vertical line in the complex $\omega$-plane to the right of all singularities of the transform functions to satisfy causality.

Taking transforms of $(2.13),(2.14)$ gives

$$
\begin{gathered}
i k U_{a}^{\dagger \dagger}+\frac{\partial V_{a}^{\dagger \dagger}}{\partial Y}=0 \\
(i k Y+\omega) U_{a}^{\dagger \dagger}+V_{a}^{\dagger \dagger}=-i k P_{a}^{\dagger \dagger}+\frac{\partial^{2} U_{a}^{\dagger \dagger}}{\partial Y^{2}} . \\
(i k Y+\omega) \theta_{a}^{\dagger \dagger}=\frac{1}{\operatorname{Pr}} \frac{\partial^{2} \theta_{a}^{\dagger \dagger}}{\partial Y^{2}} .
\end{gathered}
$$

The equation $(2.16 c)$ for the temperature perturbation may be solved in terms of Airy functions to obtain the solution

$$
\theta_{a}^{\dagger \dagger}=D_{0} \operatorname{Ai}\left(\operatorname{Pr}^{1 / 3} \xi\right)+D_{1} \operatorname{Bi}\left(\operatorname{Pr}^{1 / 3} \xi\right)
$$

where

$$
\xi=(i k)^{1 / 3} Y+\xi_{0}, \quad \xi_{0}=\omega(i k)^{-2 / 3} .
$$

We will take a branch cut along the positive imaginary axis so that $-3 \pi / 2<\arg (k)<$ $\pi / 2$. Then the function $\operatorname{Bi}(\xi)$ grows exponentially when $Y \rightarrow \infty$ and hence $D_{1}$ must be zero. Application of the boundary conditions yields

$$
\theta_{a}^{\dagger \dagger}=g^{\dagger \dagger}(k, \omega) \frac{\operatorname{Ai}\left(P r^{1 / 3} \xi\right)}{\operatorname{Ai}\left(\eta_{0}\right)}
$$

where we have written

$$
\eta_{0}=\operatorname{Pr}^{1 / 3} \xi_{0}
$$

Next differentiating $(2.16 b)$ with respect to $Y$ and using the continuity equation shows that

This has the solution

$$
\frac{\partial^{3} U_{a}^{\dagger \dagger}}{\partial Y^{3}}-(i k Y+\omega) \frac{\partial U_{a}^{\dagger \dagger}}{\partial Y}=0
$$

$$
\frac{\partial U_{a}^{\dagger \dagger}}{\partial Y}=C_{0} \operatorname{Ai}(\xi)+C_{1} \operatorname{Bi}(\xi)
$$

The Airy function $\operatorname{Bi}(\xi)$ grows exponentially for large $Y$ and so we must take $C_{1}=0$. Setting $Y=0$ in $(2.16 b)$ and using (2.21) gives

$$
(i k)^{1 / 3} C_{0} \operatorname{Ai}^{\prime}\left(\xi_{0}\right)=i k P_{a}^{\dagger \dagger} .
$$

We can further integrate (2.21) to obtain

$$
U_{a}^{\dagger \dagger}=C_{0}(i k)^{-\frac{1}{3}} \int_{\xi_{0}}^{\xi} \operatorname{Ai}(\xi) d \xi
$$


Letting $Y \rightarrow \infty$ in (2.23) and using the transformed boundary conditions from (2.14) shows that

$$
K_{a}^{\dagger \dagger}=C_{0}(i k)^{-\frac{1}{3}} \int_{\xi_{0}}^{\infty} \operatorname{Ai}(\xi) d \xi
$$

We also have from $(2.13 e)$

$$
K_{a}^{\dagger \dagger}=A_{a}^{\dagger \dagger}+\int_{0}^{\infty} \theta_{a}^{\dagger \dagger} d Y+\frac{h^{\dagger}(k) \omega_{0}}{\left(\omega^{2}+\omega_{0}^{2}\right)}
$$

which after using the solution for $\theta_{a}^{\dagger \dagger}$ becomes

$$
K_{a}^{\dagger \dagger}=A_{a}^{\dagger \dagger}+g^{\dagger \dagger}(i k P r)^{-1 / 3} \int_{\eta_{0}}^{\infty} \frac{\operatorname{Ai}(\eta)}{\operatorname{Ai}\left(\eta_{0}\right)} d \eta+\frac{h^{\dagger}(k) \omega_{0}}{\left(\omega^{2}+\omega_{0}^{2}\right)} .
$$

The equations for $P_{u}$ do not involve $\tau$ explicitly and therefore taking Fourier-Laplace transforms of $(2.15 a)$ and applying the boundary conditions gives the usual relation,

$$
P_{a}^{\dagger \dagger}=\frac{k^{2}}{|k|} A_{a}^{\dagger \dagger}
$$

Finally eliminating $C_{0}$ and solving for $P_{a}^{\dagger \dagger}$ from $(2.22),(2.24),(2.27)$ gives

$$
P_{a}^{\dagger \dagger}(k, \omega)=P_{ \pm}^{\dagger \dagger}(\omega, k)=\frac{H^{\dagger \dagger}(k, \omega) \omega_{0}|k| \mathrm{Ai}^{\prime}\left(\xi_{0}\right)}{\left(\omega^{2}+\omega_{0}^{2}\right) D^{ \pm}\left(\xi_{0}, k\right)},
$$

where $\xi_{0}$ is defined in $(2.18)$ and

$$
D^{ \pm}\left(\xi_{0}, k\right)=-\mathrm{Ai}^{\prime}\left(\xi_{0}\right) \pm k(i k)^{\frac{1}{3}} \int_{\xi_{0}}^{\infty} \operatorname{Ai}(\xi) d \xi
$$

with the plus sign in $D^{ \pm}$corresponding to $k$ positive and the minus sign for $k$ negative. In the above expression we have defined

$$
H^{\dagger \dagger}(k, \omega)=h^{\dagger}(k)+g^{\dagger \dagger}(k, \omega) \frac{\left(\omega^{2}+\omega_{0}^{2}\right)(i k P r)^{-1 / 3} \int_{\eta_{0}}^{\infty} \operatorname{Ai}(\eta) d \eta}{\omega_{0} \operatorname{Ai}\left(\eta_{0}\right)} .
$$

The disturbed pressure $P_{a}(\tau, X)$ is calculated by formally inverting (2.28).

A number of results are immediately apparent from (2.28). Notice that if we have no localised heating and set $g^{\dagger \dagger}$ to be zero, then (2.28) reduces to the expression obtained by Terent'ev (1984). Somewhat more interesting is that, even without a vibrator, localised heating is also able to excite Tollmien-Schlichting waves (as also discussed below). With a vibrator present, if the localised heating profile is chosen such that $H^{\dagger \dagger}=0$ then the response $P_{a}^{\dagger \dagger}$ is zero, which means no Tollmien-Schlichting waves. In fact the required localised heating profile is given by $g^{\dagger \dagger}(\omega, k)=g_{T C}^{\dagger \dagger}$ where

$$
g_{T C}^{\dagger \dagger}(\omega, k)=-\frac{h^{\dagger}(k) \omega_{0} \operatorname{Ai}\left(\eta_{0}\right)(i k P r)^{1 / 3}}{\left(\omega^{2}+\omega_{0}^{2}\right) \int_{\eta_{0}}^{\infty} \operatorname{Ai}(\eta) d \eta} .
$$

The expression (2.31) is significant and potentially gives a means by which it is possible to control instabilities in the boundary layer. Some typical results emanating from this formula are shown later in the paper.

2.2. Inverse transform to find the pressure $P_{a}(\tau, X)$.

Applying the inverse transform to $P_{a}^{\dagger \dagger}$ given by (2.28) shows that 


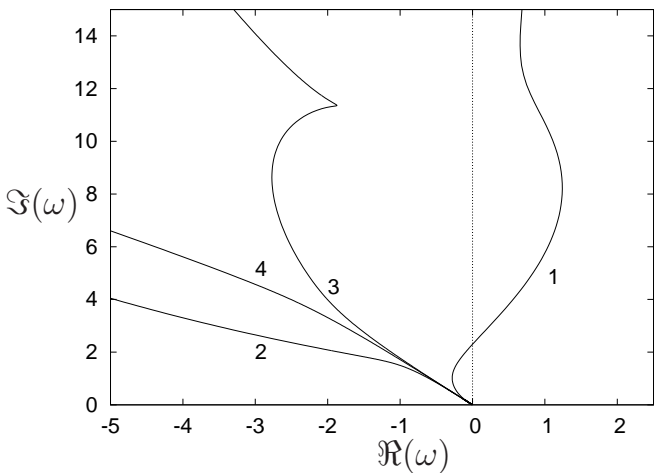

(a)

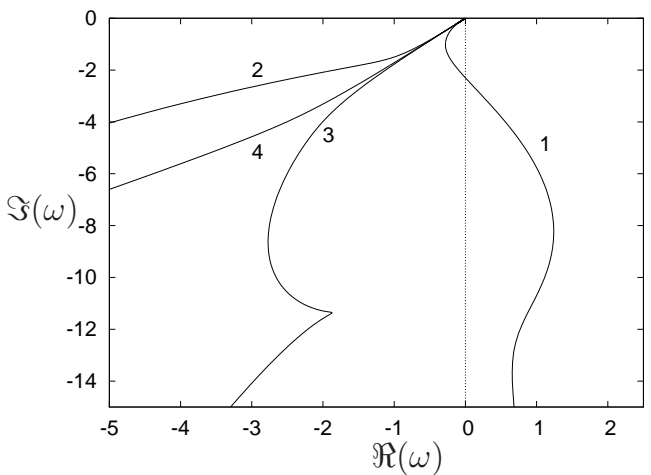

(b)

Figure 3: Locus of the (a) roots $\omega_{1, j}$ of $D^{-}\left(\xi_{0}, k\right)=0$ for $k$ varying from $(-\infty, 0)$ and (b) $\omega_{2, j}$ of $D^{+}\left(\xi_{0}, k\right)=0$ with $k$ varying from $(0, \infty)$. The labels correspond to the different $\operatorname{roots} \omega_{1, j}, \omega_{2, j}, \quad j=1,2, \ldots$

$$
\begin{aligned}
P_{a}(\tau, X)=P(\tau, X)=\frac{1}{4 \pi^{2} i} & {\left[\int_{-\infty}^{0} \int_{\mathcal{L}} P_{-}^{\dagger \dagger}(k, \omega) e^{i k X+\omega \tau} d \omega d k\right.} \\
+ & \left.\int_{0}^{\infty} \int_{\mathcal{L}} P_{+}^{\dagger \dagger}(k, \omega) e^{i k X+\omega \tau} d \omega d k\right] .
\end{aligned}
$$

We will further write

$$
P_{ \pm}^{\dagger \dagger}=P_{ \pm}^{(V) \dagger \dagger}+P_{ \pm}^{(H) \dagger \dagger}
$$

with

$$
P_{ \pm}^{(V) \dagger \dagger}=\frac{h^{\dagger}(k) \omega_{0}|k| \operatorname{Ai}^{\prime}\left(\xi_{0}\right)}{\left(\omega^{2}+\omega_{0}^{2}\right) D^{ \pm}\left(\xi_{0}, k\right)}, \quad P_{ \pm}^{(H) \dagger \dagger}=\frac{\operatorname{Ai}^{\prime}\left(\xi_{0}\right)|k|(i k P r)^{-1 / 3} g^{\dagger \dagger}(k, \omega) \int_{\eta_{0}}^{\infty} \operatorname{Ai}(\eta) d \eta}{\operatorname{Ai}\left(\eta_{0}\right) D^{ \pm}\left(\xi_{0}, k\right)}
$$

The superscripts $(V)$ and $(H)$ separate out the effects due to the vibrator and localised heating. Consider first

$$
P_{-}^{(V)}(\tau, X)=\frac{1}{4 \pi^{2} i} \int_{-\infty}^{0} \int_{\mathcal{L}} P_{-}^{(V) \dagger \dagger}(k, \omega) e^{i k X+\omega \tau} d \omega d k
$$

\subsection{Inversion of the pressure $P_{-}^{(V)}$ the vibrator contribution}

In the complex $\omega$-plane the integrand has poles at $\omega= \pm i \omega_{0}$ and at the zeros of $D^{-}\left(\xi_{0}, k\right)=0$. In Figure 3(a) the first few zeros are shown for $k$ varying from $-\infty$ to zero. Suppose that we label the roots $\omega_{1, j}, \quad(j=1,2, \ldots)$ then for $j=2,3, \ldots$ the roots have the property that $\Re\left(\omega_{1, j}\right)<0$. Only the root $\omega_{1,1}$ crosses the imaginary axis into the first quadrant when $k=k^{*}$ and with $\omega_{1,1}\left(k_{*}\right)=i \omega^{*}$. The properties of the roots of the dispersion relation $D^{ \pm}\left(\xi_{0}, k\right)=0$ have been studied in many papers including Terent'ev (1981, 1984), Walker et al. (2006), and Ruban et al. (2016). In order to evaluate the inner integral in (2.36) we deform the contour $\mathcal{L}$ into the left hand $\omega$-plane as shown in Figure 4. Using Cauchy's theorem

$$
\frac{1}{2 \pi i}\left(\int_{L}+\int_{C_{1}+C_{2}+C_{R}}\right) P_{-}^{(V) \dagger \dagger} e^{i k X+\omega \tau} d \omega
$$

$=\sum\left[\right.$ Residues of $P_{-}^{(V) \dagger \dagger} e^{i k X+\omega \tau} \quad$ inside the contour $\left.\mathcal{L}+C_{1}+C_{2}+C_{R}\right] .(2.37)$ 


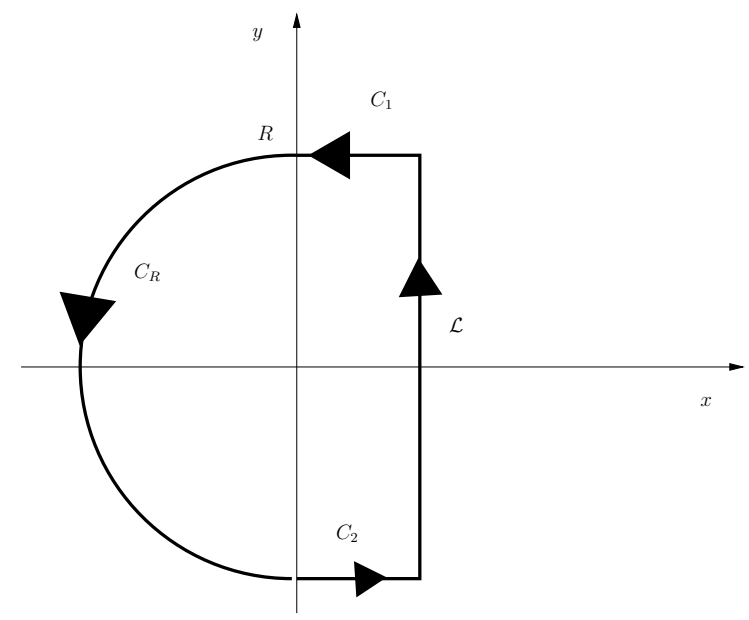

Figure 4: Deformed contour for inversion of the integral in the $\omega$-plane.

The residues arise from poles at $\omega= \pm i \omega_{0}$ and when $\omega=\omega_{1, j}$. Hence

$$
\begin{aligned}
& \frac{1}{2 \pi i}\left(\int_{L}+\int_{C_{1}+C_{2}+C_{R}}\right) P_{-}^{(V) \dagger \dagger} e^{i k X+\omega \tau} d \omega=\frac{|k| h^{\dagger}(k) \operatorname{Ai}^{\prime}\left(\xi_{0}\left(i \omega_{0}, k\right)\right)}{2 i D^{-}\left(\xi_{0}\left(i \omega_{0}, k\right), k\right)} e^{i \omega_{0} \tau+i k X} \\
- & \frac{|k| h^{\dagger}(k) \operatorname{Ai}^{\prime}\left(\xi_{0}\left(-i \omega_{0}, k\right)\right)}{2 i D^{-}\left(\xi_{0}\left(-i \omega_{0}, k\right), k\right)} e^{-i \omega_{0} \tau+i k X}+\sum_{j} \frac{|k| \omega_{0} h^{\dagger}(k) \operatorname{Ai}^{\prime}\left(\xi_{0}\left(\omega_{1, j}, k\right)\right)}{\left(\omega_{0}^{2}+\omega_{1, j}^{2}\right) \frac{\partial D^{-}}{\partial \omega}\left(\xi_{0}\left(\omega_{1, j}, k\right), k\right)} e^{\omega_{1, j} \tau+i k X} .
\end{aligned}
$$

The integrals $\int_{C_{1}}, \int_{C_{2}}, \int_{C_{R}}$ can be shown to tend to zero when $R \rightarrow \infty$. Hence

$$
\begin{aligned}
P_{-}^{(V)}(\tau, X) & =\frac{1}{2 \pi} \int_{-\infty}^{0} \frac{|k| h^{\dagger}(k) \mathrm{Ai}^{\prime}\left(\xi_{0}\left(i \omega_{0}, k\right)\right)}{2 i D^{-}\left(\xi_{0}\left(i \omega_{0}, k\right), k\right)} e^{i \omega_{0} \tau+i k X} d k \\
& -\frac{1}{2 \pi} \int_{-\infty}^{0} \frac{|k| h^{\dagger}(k) \mathrm{Ai}^{\prime}\left(\xi_{0}\left(-i \omega_{0}, k\right)\right)}{2 i D^{-}\left(\xi_{0}\left(-i \omega_{0}, k\right), k\right)} e^{-i \omega_{0} \tau+i k X} d k \\
& +\frac{1}{2 \pi} \sum_{j} \int_{-\infty}^{0} \frac{|k| \omega_{0} h^{\dagger}(k) \mathrm{Ai}^{\prime}\left(\xi_{0}\left(\omega_{1, j}, k\right)\right)}{\left(\omega_{0}^{2}+\omega_{1, j}^{2}\right) \frac{\partial D^{-}}{\partial \omega}\left(\xi_{0}\left(\omega_{1, j}, k\right), k\right)} e^{\omega_{1, j}(k) \tau+i k X} d k
\end{aligned}
$$

The above calculation can be repeated for the inversion of $P_{+}^{(V) \dagger \dagger}$ and the details are very similar to those described above and give

$$
\begin{aligned}
P_{+}^{(V)}(\tau, X) & =\frac{1}{2 \pi} \int_{0}^{\infty} \frac{|k| h^{\dagger}(k) \mathrm{Ai}^{\prime}\left(\xi_{0}\left(i \omega_{0}, k\right)\right)}{2 i D^{+}\left(\xi_{0}\left(i \omega_{0}, k\right), k\right)} e^{i \omega_{0} \tau+i k X} d k \\
& -\frac{1}{2 \pi} \int_{0}^{\infty} \frac{|k| h^{\dagger}(k) \mathrm{Ai}^{\prime}\left(\xi_{0}\left(-i \omega_{0}, k\right)\right)}{2 i D^{+}\left(\xi_{0}\left(-i \omega_{0}, k\right), k\right)} e^{-i \omega_{0} \tau+i k X} d k \\
& +\frac{1}{2 \pi} \sum_{j} \int_{0}^{\infty} \frac{|k| \omega_{0} h^{\dagger}(k) \mathrm{Ai}^{\prime}\left(\xi_{0}\left(\omega_{2, j}, k\right)\right)}{\left(\omega_{0}^{2}+\omega_{2, j}^{2}\right) \frac{\partial D^{+}}{\partial \omega}\left(\xi_{0}\left(\omega_{2, j}, k\right), k\right)} e^{\omega_{2, j}(k) \tau+i k X} d k
\end{aligned}
$$

In (2.39) we have labelled $\omega_{2, j}(k)$ as the zeros of $D^{+}\left(\xi_{0}, k\right)=0$ for $k$ positive. 


\subsection{Inversion of the pressure $P_{-}^{(H)}$ the localised heating contribution}

Since $P_{-}^{(H)}$ involves the function $g^{\dagger \dagger}(\omega, k)$ many profiles could be chosen but suppose we take a localised heating profile of the form

$$
g(\tau, x)=\hat{g}(x) \sin \left(\omega_{0} \tau\right)
$$

giving

Then

$$
g^{\dagger \dagger}(\omega, k)=\frac{\hat{g}^{\dagger}(k) \omega_{0}}{\omega^{2}+\omega_{0}^{2}}
$$

$$
P_{ \pm}^{(H) \dagger \dagger}=\frac{A i^{\prime}\left(\xi_{0}\right)|k|(i k P r)^{-1 / 3} \hat{g}^{\dagger}(k) \omega_{0} \int_{\eta_{0}}^{\infty} \operatorname{Ai}(\eta) d \eta}{\left(\omega^{2}+\omega_{0}^{2}\right) \operatorname{Ai}\left(\eta_{0}\right) D^{ \pm}\left(\xi_{0}, k\right)} .
$$

In inverting the transform $P_{ \pm}^{(H) \dagger \dagger}$ in addition to the poles discussed when inverting $P_{ \pm}^{(V) \dagger \dagger}$ we also have additional poles, which we label as $\omega=\omega_{3, j}(k), \omega_{4, j}(k)$, arising from the zeros of $\operatorname{Ai}\left(\eta_{0}\right)$ in the denominator of (2.40). The Airy function $\operatorname{Ai}\left(\eta_{0}\right)$ has zeros on the negative real axis and we can write

$$
\eta_{0}=-\left|a_{j}\right| \quad(j=1,2, \ldots)
$$

where $a_{j}$ is a zero of the Airy function $\operatorname{Ai}(\eta)$. Using the definition of $\eta_{0}$ from (2.18) and (2.20) we obtain

$$
\omega(k)=\omega_{3, j}=\frac{\left|a_{j} k^{\frac{2}{3}}\right|}{\operatorname{Pr}^{1 / 3}} e^{-4 i \pi / 3} \quad(k<0),
$$

and

$$
\omega(k)=\omega_{4, j}=\frac{\left|a_{j} k^{\frac{2}{3}}\right|}{\operatorname{Pr}^{1 / 3}} e^{-2 i \pi / 3} \quad(k>0) .
$$

Since the real part of $\omega_{3, j}(k), \omega_{4, j}(k)$ is negative these do not contribute to any additional unstable modes. The inversion follows a similar argument to that already given and it can be shown that

$$
\begin{aligned}
& 2 \pi P_{-}^{(H)}(\tau, X)=\int_{-\infty}^{0} \frac{\hat{g}^{\dagger}(k)|k|^{\frac{2}{3}} e^{\frac{i \pi}{6}}(P r)^{-\frac{1}{3}} \operatorname{Ai}^{\prime}\left(\xi_{0}\left(i \omega_{0}, k\right)\right) \int_{\eta_{0}\left(i \omega_{0}, k\right)}^{\infty} \operatorname{Ai}(\eta) d \eta}{2 i \operatorname{Ai}\left(\eta_{0}\left(i \omega_{0}, k\right)\right) D^{-}\left(\xi_{0}\left(i \omega_{0}, k\right), k\right)} e^{i \omega_{0} \tau+i k X} d k \\
& -\int_{-\infty}^{0} \frac{\hat{g}^{\dagger}(k)|k|^{\frac{2}{3}} e^{\frac{i \pi}{6}}(P r)^{-\frac{1}{3}} \operatorname{Ai}^{\prime}\left(\xi_{0}\left(-i \omega_{0}, k\right)\right) \int_{\eta_{0}\left(-i \omega_{0}, k\right)}^{\infty} \operatorname{Ai}(\eta) d \eta}{2 i \operatorname{Ai}\left(\eta\left(-i \omega_{0}, k\right)\right) D^{-}\left(\xi_{0}\left(-i \omega_{0}, k\right), k\right)} e^{-i \omega_{0} \tau+i k X} d k \\
& +\sum_{j} \int_{-\infty}^{0} \frac{\omega_{0} \hat{g}^{\dagger}(k)|k|^{\frac{2}{3}} e^{\frac{i \pi}{6}}(P r)^{-\frac{1}{3}} \operatorname{Ai}^{\prime}\left(\xi_{0}\left(\omega_{1, j}, k\right)\right) \int_{\eta_{0}\left(\omega_{1, j}, k\right)}^{\infty} \operatorname{Ai}(\eta) d \eta}{\left(\omega_{0}^{2}+\omega_{1, j}^{2}\right) \operatorname{Ai}\left(\eta_{0}\left(\omega_{1, j}, k\right)\right) \frac{\partial D^{-}}{\partial \omega}\left(\xi_{0}\left(\omega_{1, j}, k\right), k\right)} e^{\omega_{1, j}(k) \tau+i k X} d k \\
& +\sum_{j} \int_{-\infty}^{0} \frac{\omega_{0} \hat{g}^{\dagger}(k) \mathrm{Ai}^{\prime}\left(\xi_{0}\left(\omega_{3, j}, k\right)\right)|k|^{\frac{4}{3}} e^{-\frac{i \pi}{6}}(P r)^{-\frac{2}{3}} \int_{\eta_{0}\left(\omega_{3, j}, k\right)}^{\infty} \operatorname{Ai}(\eta) d \eta}{\left(\omega_{0}^{2}+\omega_{3, j}^{2}\right) \mathrm{Ai}^{\prime}\left(\eta_{0}\left(\omega_{3, j}, k\right)\right) D^{-}\left(\xi_{0}\left(\omega_{3, j}, k\right), k\right)} e^{\omega_{3, j}(k) \tau+i k X} d k .
\end{aligned}
$$

An expression for $P_{+}^{(H)}$ may be derived in a similar manner.

Finally

$$
P(\tau, X)=P_{-}(\tau, X)+P_{+}(\tau, X) .
$$

To interpret the above findings we need the following additional results. 
First note that if we write $k=|k| e^{i \theta_{k}}$ where $\theta_{k}$ is the argument of $k$ then $\xi_{0}(\omega, k)$ can be written as

$$
\xi_{0}(\omega, k)=\omega(i k)^{-2 / 3}=\omega|k|^{-2 / 3} e^{-2 i \theta_{k} / 3} e^{-i \pi / 3} .
$$

Hence the complex conjugate of $\xi_{0}(\omega, k)$ is

$$
\left[\xi_{0}(\omega, k)\right]^{(c)}=\omega^{(c)}|k|^{-2 / 3} e^{2 i \theta_{k} / 3} e^{i \pi / 3} .
$$

But note that

$$
\xi_{0}\left(\omega^{(c)}, k^{(c)} e^{-i \pi}\right)=\omega^{(c)}|k|^{-2 / 3} e^{2 i\left(\theta_{k}+\pi\right) / 3} e^{-i \pi / 3}=\omega^{(c)}|k|^{-2 / 3} e^{2 i \theta_{k} / 3} e^{i \pi / 3} .
$$

This shows that

$$
\left[\xi_{0}(\omega, k)\right]^{(c)}=\xi_{0}\left(\omega^{(c)}, k^{(c)} e^{-i \pi}\right) .
$$

Using this we observe that if $D^{-}\left(\xi_{0}(\omega, k)\right)=0$ then taking the complex conjugate

$$
\begin{aligned}
{\left[D^{-}\left(\xi_{0}(\omega, k), k\right)\right]^{(c)} } & =-\operatorname{Ai}^{\prime}\left(\xi_{0}^{(c)}\right)-|k|^{4 / 3} e^{-\frac{4}{3} i \theta_{k}} e^{-i \pi / 6} \int_{\xi_{0}^{(c)}}^{\infty} \operatorname{Ai}(\xi) d \xi \\
& =-\operatorname{Ai}^{\prime}\left(\xi_{0}^{(c)}\right)+|k| e^{i \frac{4}{3}\left(-\pi-\theta_{k}\right)} e^{i \pi / 6} \int_{\xi_{0}^{(c)}}^{\infty} \operatorname{Ai}(\xi) d \xi \\
& =D^{+}\left(\xi_{0}\left(\omega^{(c)}, k^{(c)} e^{-i \pi}\right), k^{(c)} e^{-i \pi}\right) .
\end{aligned}
$$

So if $(\omega, k)$ is a root of $D^{-}=0$ then $\left(\omega^{(c)}, k^{(c)} e^{-i \pi}\right)$ is a root of $D^{+}=0$; figure 3 confirms this observation. We can use these results to rewrite the inverted pressure as

$$
\begin{aligned}
& P(\tau, X)=\frac{1}{\pi} \Re\left[\int_{-\infty}^{0} \frac{|k| h^{\dagger}(k) \mathrm{Ai}^{\prime}\left(\xi_{0}\left(i \omega_{0}, k\right)\right)}{2 i D^{-}\left(\xi_{0}\left(i \omega_{0}, k\right), k\right)} e^{i \omega_{0} \tau+i k X} d k\right. \\
& -\int_{-\infty}^{0} \frac{|k| h^{\dagger}(k) \mathrm{Ai}^{\prime}\left(\xi_{0}\left(-i \omega_{0}, k\right)\right)}{2 i D^{-}\left(\xi_{0}\left(-i \omega_{0}, k\right), k\right)} e^{-i \omega_{0} \tau+i k X} d k \\
& +\sum_{j} \int_{-\infty}^{0} \frac{|k| \omega_{0} h^{\dagger}(k) \mathrm{Ai}^{\prime}\left(\xi_{0}\left(\omega_{1, j}, k\right)\right)}{\left(\omega_{0}^{2}+\omega_{1, j}^{2}\right) \frac{\partial D^{-}}{\partial \omega}\left(\xi_{0}\left(\omega_{1, j}, k\right), k\right)} e^{\omega_{1, j}(k) \tau+i k X} d k \\
& +\int_{-\infty}^{0} \frac{\hat{g}^{\dagger}(k)|k|^{\frac{2}{3}} e^{\frac{i \pi}{6}}(\operatorname{Pr})^{-\frac{1}{3}} \mathrm{Ai}^{\prime}\left(\xi_{0}\left(i \omega_{0}, k\right)\right) \int_{\eta_{0}\left(i \omega_{0}, k\right)}^{\infty} \operatorname{Ai}(\eta) d \eta}{2 i \mathrm{Ai}\left(\eta_{0}\left(i \omega_{0}, k\right)\right) D^{-}\left(\xi_{0}\left(i \omega_{0}, k\right), k\right)} e^{i \omega_{0} \tau+i k X} d k \\
& -\int_{-\infty}^{0} \frac{\hat{g}^{\dagger}(k)|k|^{\frac{2}{3}} e^{\frac{i \pi}{6}}(\operatorname{Pr})^{-\frac{1}{3}} \mathrm{Ai}^{\prime}\left(\xi_{0}\left(-i \omega_{0}, k\right)\right) \int_{\eta_{0}\left(-i \omega_{0}, k\right)}^{\infty} \mathrm{Ai}(\eta) d \eta}{2 i \mathrm{Ai}\left(\eta\left(-i \omega_{0}, k\right)\right) D^{-}\left(\xi_{0}\left(-i \omega_{0}, k\right), k\right)} e^{-i \omega_{0} \tau+i k X} d k \\
& +\sum_{j} \int_{-\infty}^{0} \frac{\omega_{0} \hat{g}^{\dagger}(k)|k|^{\frac{2}{3}} e^{\frac{i \pi}{6}}(\operatorname{Pr})^{-\frac{1}{3}} \mathrm{Ai}^{\prime}\left(\xi_{0}\left(\omega_{1, j}, k\right)\right) \int_{\eta_{0}\left(\omega_{1, j}, k\right)}^{\infty} \mathrm{Ai}(\eta) d \eta}{\left(\omega_{0}^{2}+\omega_{1, j}^{2}\right) \mathrm{Ai}^{\prime}\left(\eta_{0}\left(\omega_{1, j}, k\right)\right) \frac{\partial D^{-}\left(\xi_{0}\left(\omega_{1, j}, k\right), k\right)}{\partial \omega} e^{\omega_{1, j}(k) \tau+i k X} d k} \\
& \left.+\sum_{j} \int_{-\infty}^{0} \frac{\omega_{0} \hat{g}^{\dagger}(k)|k|^{\frac{4}{3}} e^{-\frac{i \pi}{6}}(\operatorname{Pr})^{-\frac{2}{3}} \mathrm{Ai}^{\prime}\left(\xi_{0}\left(\omega_{3, j}, k\right)\right) \int_{\eta_{0}\left(\omega_{3, j}, k\right)}^{\infty} \operatorname{Ai}(\eta) d \eta}{\left(\omega_{0}^{2}+\omega_{3, j}^{2}\right) \mathrm{Ai}^{\prime}\left(\eta_{0}\left(\omega_{3, j}, k\right)\right) D^{-}\left(\xi_{0}\left(\omega_{3, j}, k\right), k\right)} e^{\omega_{3, j}(k) \tau+i k X} d k\right]
\end{aligned}
$$

Notice that the integrals in (2.42) (with terms multiplied by $e^{ \pm i \omega_{0} \tau}$ ) represent the response due to the forcing at the vibrator frequency. The other terms are the response due to the excited modes of the boundary layer. For large time $\tau$ all the terms in the summations except the one with $j=1$ corresponding to the root $\omega_{1, j}$ decay rapidly. If $j=1$ the integrands proportional to $e^{\omega_{1, j} \tau}$ have a simple pole at $k=k_{1}$ when $\omega_{1,1}\left(k_{1}\right)=$ 
$i \omega_{0}$. The locus of the root $\omega_{1,1}(k)=i \omega_{0}$ in the complex $k$-plane for varying $\omega_{0}$ is shown in figure 5. When $\omega_{0}=\omega^{*}=2.298$ and $k=k^{*}=-1.005$ the root crosses from the second quadrant into the third-quadrant in the $k$-plane giving rise to unstable waves. In fact we can estimate the terms by making use of Laplace's method and noting that the major contribution to the integrals come from the vicinity of $k=0$. The details of the lengthy calculation are omitted but are similar to those given by Terent'ev (1984), Ruban et al. (2016) for instance. It is found that for large $\tau$ we can approximate (2.42) by

$$
\begin{aligned}
& P(\tau, X)=\Re\left[\frac{1}{\pi} \int_{-\infty}^{0} \frac{|k| h^{\dagger}(k) \mathrm{Ai}^{\prime}\left(\xi_{0}\left(i \omega_{0}, k\right)\right)}{2 i D^{-}\left(\xi_{0}\left(i \omega_{0}, k\right), k\right)} e^{i \omega_{0} \tau+i k X} d k\right. \\
& -\frac{1}{\pi} \int_{-\infty}^{0} \frac{|k| h^{\dagger}(k) \mathrm{Ai}^{\prime}\left(\xi_{0}\left(-i \omega_{0}, k\right)\right)}{2 i D^{-}\left(\xi_{0}\left(-i \omega_{0}, k\right), k\right)} e^{-i \omega_{0} \tau+i k X} d k \\
& +\frac{1}{\pi} \int_{-\infty}^{0} \frac{\hat{g}^{\dagger}(k) \operatorname{Ai}^{\prime}\left(\xi_{0}\left(i \omega_{0}, k\right)\right)|k|^{\frac{2}{3}} e^{\frac{i \pi}{6}}(\operatorname{Pr})^{-\frac{1}{3}} \int_{\eta_{0}\left(i \omega_{0}, k\right)}^{\infty} \operatorname{Ai}(\eta) d \eta}{2 i \operatorname{Ai}\left(\eta_{0}\left(i \omega_{0}, k\right)\right) D^{-}\left(\xi_{0}\left(i \omega_{0}, k\right), k\right)} e^{i \omega_{0} \tau+i k X} d k \\
& -\frac{1}{\pi} \int_{-\infty}^{0} \frac{\hat{g}^{\dagger}(k) \operatorname{Ai}^{\prime}\left(\xi_{0}\left(-i \omega_{0}, k\right)\right)|k|^{\frac{2}{3}} e^{\frac{i \pi}{6}}(\operatorname{Pr})^{-\frac{1}{3}} \int_{\eta_{0}\left(-i \omega_{0}, k\right)}^{\infty} \operatorname{Ai}(\eta) d \eta}{2 i \operatorname{Ai}\left(\eta\left(-i \omega_{0}, k\right)\right) D^{-}\left(\xi_{0}\left(-i \omega_{0}, k\right), k\right)} e^{-i \omega_{0} \tau+i k X} d k \\
& -\frac{\left|k_{1}\right| h^{\dagger}\left(k_{1}\right) \mathrm{Ai}^{\prime}\left(\xi_{0}\left(i \omega_{0}, k_{1}\right)\right)}{\frac{\partial D^{-}}{\partial \omega}\left(\xi_{0}\left(i \omega_{0}, k_{1}\right), k_{1}\right) \frac{d \omega_{1,1}}{d k}\left(k_{1}\right)} e^{\omega_{1,1}\left(k_{1}\right) \tau+i k_{1} X} \mathcal{H}\left(\omega_{0}-\omega^{*}\right) \\
& \left.-\frac{\hat{g}^{\dagger}\left(k_{1}\right)|k|^{\frac{2}{3}} e^{\frac{i \pi}{6}}(P r)^{-\frac{1}{3}} \operatorname{Ai}^{\prime}\left(\xi_{0}\left(i \omega_{0}, k_{1}\right)\right) \int_{\eta_{0}\left(i \omega_{0}, k_{1}\right)}^{\infty} \operatorname{Ai}(\eta) d \eta}{\operatorname{Ai}\left(\eta_{0}\left(i \omega_{0}, k_{1}\right)\right) \frac{\partial D^{-}}{\partial \omega}\left(\xi_{0}\left(i \omega_{0}, k_{1}\right), k_{1}\right) \frac{d \omega_{1, j}}{d k}\left(k_{1}\right)} e^{\omega_{1,1}\left(k_{1}\right) \tau+i k_{1} X} \mathcal{H}\left(\omega_{0}-\omega^{*}\right),\right]
\end{aligned}
$$

where $\mathcal{H}(\theta)$ is the Heaviside function. The result (2.43) shows that for frequencies larger than the critical frequency $\omega^{*}=2.298$ the vibrator as as well localised heating is able to excite an unstable wave in the boundary layer whose shape and amplitude is given by the last two terms in (2.43). If we write the expression for the unstable wave in the form

$$
J\left(\omega_{0}, k_{1}\right) e^{\omega_{1,1}\left(k_{1}\right) \tau+i k_{1} X}
$$

then

$$
J\left(\omega_{0}, k_{1}\right)=J_{V}\left(\omega_{0}, k_{1}\right)+J_{H}\left(\omega_{0}, k_{1}\right)
$$

with

$$
\begin{aligned}
& J_{V}\left(\omega_{0}, k_{1}\right)=-\Re\left[\frac{\left|k_{1}\right| h^{\dagger}\left(k_{1}\right) \mathrm{Ai}^{\prime}\left(\xi_{0}\left(i \omega_{0}, k_{1}\right)\right)}{\frac{\partial D^{-}}{\partial \omega}\left(\xi_{0}\left(i \omega_{0}, k_{1}\right), k_{1}\right) \frac{d \omega_{1,1}}{d k}\left(k_{1}\right)}\right] \\
& J_{H}\left(\omega_{0}, k_{1}\right)=-\Re\left[\frac{\hat{g}^{\dagger}\left(k_{1}\right)|k|^{2 / 3} e^{\frac{i \pi}{6}}(P r)^{-1 / 3} \operatorname{Ai}^{\prime}\left(\xi_{0}\left(i \omega_{0}, k_{1}\right)\right) \int_{\eta_{0}\left(i \omega_{0}, k_{1}\right)}^{\infty} \operatorname{Ai}(\eta) d \eta}{\operatorname{Ai}\left(\eta_{0}\left(i \omega_{0}, k_{1}\right)\right) \frac{\partial D^{-}}{\partial \omega}\left(\xi_{0}\left(i \omega_{0}, k_{1}\right), k_{1}\right) \frac{\omega_{1,1}}{d k}\left(k_{1}\right)}\right] .
\end{aligned}
$$

The terms $J_{V}, J_{H}$ in (2.44) give the respective amplitudes of the generated TollmienSchlichting wave due to the vibrator and heating respectively. 


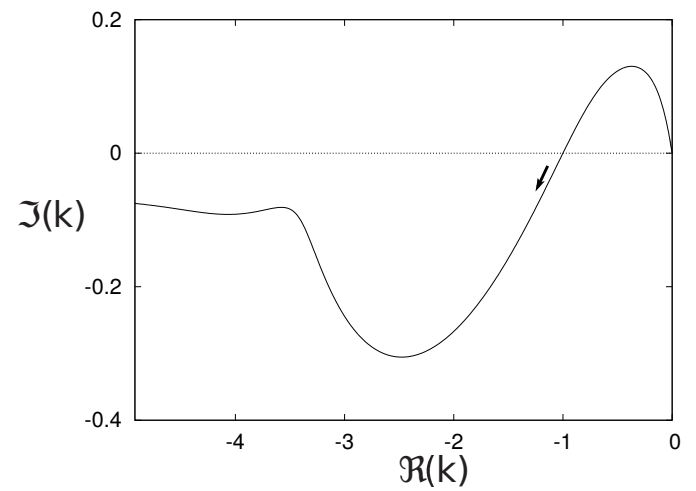

Figure 5: Locus of the root $\omega_{1,1}(k)=i \omega_{0}$ with $\omega_{0}$ varying from 0 to $\infty$. The arrow indicates the direction of $\omega_{0}$ increasing.

\section{Numerical solution of the linearized triple-deck initial-value problem}

As an alternative to the analytical solution of the previous section, here we focus on obtaining results using a numerical approach to solving the initial-value problem. Consider the linearised triple-deck problem governed by $(2.13),(2.14),(2.16)$

$$
\begin{aligned}
\frac{\partial U_{a}}{\partial X}+\frac{\partial V_{a}}{\partial Y} & =0 \\
\frac{\partial U_{a}}{\partial \tau}+Y \frac{\partial U_{a}}{\partial X}+V_{a} & =-\frac{\partial P_{a}}{\partial X}+\frac{\partial^{2} U_{a}}{\partial Y^{2}}, \\
\frac{\partial \theta_{a}}{\partial \tau}+Y \frac{\partial \theta_{a}}{\partial X} & =\frac{1}{\operatorname{Pr}} \frac{\partial^{2} \theta_{a}}{\partial Y^{2}},
\end{aligned}
$$

for $|x|<\infty, Y>0$ and $\tau>0$. The boundary and initial conditions are

$$
\begin{gathered}
U_{a}, V_{a}, \theta_{a}, P_{a}, A_{a}, K_{a}, F_{a}, \theta_{w}=0 \quad \tau<0 \\
U_{a}(\tau, X, Y=0)=V_{a}(\tau, X, Y=0)=0, \\
K_{a}(\tau, X)=F_{a}(\tau, X)+A_{a}(\tau, X)+\int_{0}^{\infty} \theta_{a}(\tau, X, Y) d Y, \quad \text { for } \tau>0, \\
U_{a}(\tau, X, Y \rightarrow \infty)=K_{a}(\tau, X), \\
U_{a}, V_{a}, P_{a}, A_{a}, \theta_{a}, K_{a}, \rightarrow 0 \quad \text { as } \quad|X| \rightarrow \infty .
\end{gathered}
$$

We also allow for more general wall-shape and heating functions, defined by

$$
F_{a}(\tau, X)=h(X) q(\tau), \quad \theta_{a w}=g(\tau, X) \quad 0<\tau .
$$

The function $h(X)$ is a smooth Gaussian hump given by $h(X)=e^{-X^{2} / 4}$. Note that this is different from the triangular shaped bump employed by Terent'ev (1984), which was given by

$$
h(X)=\left\{\begin{array}{l}
0 \quad X<0 \text { or } X>2 \\
X \text { for } 0<X<1 \\
2-X \text { for } 1<X<2
\end{array} .\right.
$$

As we shall discuss later, the use of (3.3) makes resolving the resulting flow substantially more challenging owing to the slow (algebraic) decay of the Fourier spectrum at high wave numbers. For the function $q(\tau)$ the Terent'ev (1984) work and the analytical 
results above use $q(\tau)=\sin \omega_{0} \tau$. For the numerical work we have used $q(\tau)=\sin \omega_{0} \tau$ as well as a smoother initial start given by

$$
q(\tau)=\left(1-e^{-a \tau^{2}}\right) \sin \left(\omega_{0} \tau\right),
$$

with $a=1 / 10$. The choice of the wall heating functions $g(\tau, X)$ used is discussed below.

We also have the relationship

$$
P_{a}(\tau, X)=\frac{1}{\pi} \int_{-\infty}^{\infty} \frac{1}{X-\xi} \frac{\partial A_{a}(\tau, \xi)}{\partial \xi} d \xi .
$$

To solve the set (3.1-3.4) and in particular to find the pressure $P_{a}(X, T)$ we performed a numerical study of the equations using the following method. First define a Fourier transform of $U_{a}(\tau, X, Y)$ by

$$
U_{a}^{\dagger}(\tau, k, Y)=\frac{1}{\sqrt{2 \pi}} \int_{-\infty}^{\infty} U_{a}(\tau, X, Y) e^{-i k X} d X
$$

The inverse is given by

$$
U_{a}(\tau, X, Y)=\frac{1}{\sqrt{2 \pi}} \int_{-\infty}^{\infty} U_{a}^{\dagger}(\tau, k, Y) e^{i k X} d X
$$

Then the equations (3.1-3.4) become

$$
\begin{gathered}
i k U_{a}^{\dagger}+\frac{\partial V_{a}^{\dagger}}{\partial Y}=0 \\
\frac{\partial U_{a}^{\dagger}}{\partial \tau}+i k Y U_{a}^{\dagger}+V_{a}^{\dagger}=-i k P_{a}^{\dagger}+\frac{\partial^{2} U_{a}^{\dagger}}{\partial Y^{2}} \\
\frac{\partial \theta_{a}^{\dagger}}{\partial \tau}+i k Y \theta_{a}^{\dagger}=\frac{1}{\operatorname{Pr}} \frac{\partial^{2} \theta_{a}^{\dagger}}{\partial Y^{2}} \\
U_{a}^{\dagger}(\tau, k, Y=0)=V_{a}(\tau, k, Y=0)=0, \quad \theta_{a}(\tau, k, Y=0)=g^{\dagger}(\tau, k), \\
K_{a}^{\dagger}(\tau, k)=q(\tau) h^{\dagger}(k)+A_{a}^{\dagger}(\tau, k)+\int_{0}^{\infty} \theta_{a}^{\dagger}(\tau, k, Y) d Y \quad \text { for } \quad \tau>0 . \\
U_{a}^{\dagger}(\tau, k, Y \rightarrow \infty)=K_{a}^{\dagger}(\tau, k), \\
P_{a}^{\dagger}(\tau, k)=\frac{k^{2}}{|k|} A_{a}^{\dagger}(\tau, k) .
\end{gathered}
$$

We use a second-order fully implicit time-differencing scheme with time step $d \tau$ and $(3.5 b)$ for example replaced by

$$
\frac{3 U_{a}^{\dagger(n+1)}-4 U_{a}^{\dagger(n)}+U_{a}^{\dagger(n-1)}}{2 d \tau}+i k Y U_{a}^{\dagger(n+1)}+V_{a}^{\dagger(n+1)}=-i k P_{a}^{\dagger(n+1)}+\frac{\partial^{2} U_{a}^{\dagger(n+1)}}{\partial Y^{2}},
$$

and from $(3.5 a) V_{a}^{\dagger}$ is given by

$$
V_{a}^{\dagger(n+1)}=-i k \int_{0}^{Y} U_{a}^{\dagger(n+1)} d Y
$$

Here for example $U_{a}^{\dagger(n)}=U_{a}^{\dagger}\left(\tau_{n}\right)$ where $\tau_{n}=n d \tau$. For the first time step we use a firstorder fully implicit scheme. For a given time level $n$ we solve the system of equations for $U_{a}^{\dagger}, P_{a}^{\dagger}, \theta_{a}^{\dagger}$ by using Chebychev collocation at the collocation points

$$
Y=Y_{j}=\frac{Y_{\max }}{2}\left(1-\cos \left(\frac{j \pi}{N}\right)\right), \quad j=0, \ldots, N
$$


with the approximate outer boundary $Y_{\max }$ chosen suitably. Let $\mathbf{u}^{\dagger(\mathbf{n})}=\left(u_{j}^{\dagger(n)}\right)$ with $u_{j}^{\dagger(n)}=U_{a}^{\dagger(n)}\left(Y=Y_{j}\right)$, and similarly $\boldsymbol{\theta}^{\dagger(n)}=\left(\theta_{a j}^{\dagger(n)}\right)$. Then (3.6) leads to a linear system of the form

$$
\mathbf{G}\left(\begin{array}{c}
\mathbf{u}^{\dagger(n+1)} \\
A_{a}^{\dagger(n+1)} \\
\boldsymbol{\theta}^{\dagger(n+1)}
\end{array}\right)=\mathbf{R}^{(n)},
$$

where $\mathbf{G}$ is a square matrix of order $(2 N+3)$ and $\mathbf{R}^{(n)}$ is the right-hand side of the linear system. To accommodate the boundary conditions we replace the first row of $\mathbf{G}$ with the condition from $(3.5 d)$

$$
u^{\dagger(n+1)}\left(Y_{0}\right)=0
$$

Row $(N+1)$ of $\mathbf{G}$ is replaced by the boundary condition

$$
u^{\dagger(n+1)}\left(Y_{N}\right)-A_{a}^{\dagger(n+1)}-\int_{0}^{\infty} \theta_{a}^{\dagger(n+1)} d Y=q\left(\tau_{n}\right) h^{\dagger}(k),
$$

making use of $(3.5 e)$. Row $(N+2)$ of $\mathbf{G}$ is replaced by the condition stemming from the equation

$$
\frac{\partial U_{a}^{\dagger}}{\partial Y}\left(Y=Y_{N}\right)=0
$$

Finally in row $(N+3)$ and the last row of $G$ we use the remaining conditions on $\theta^{\dagger}$ at $Y=0$ and $Y=Y_{N}$ respectively.

For a given $k$, we can solve the linear system (3.8) to find the unknown variables and in particular $P_{a}^{\dagger(n)}(k)$. This is then inverted using the discrete inverse Fast Fourier Transform to obtain $P_{a}\left(\tau_{n}, X\right)$ and other quantities as required.

\section{Results}

Results are presented at various time intervals and for different frequencies. For the numerical work we have taken the Prandtl number $\operatorname{Pr}=1$. Figure 6,7 and 8 show the pressure perturbation for $\omega=2, \quad \omega=2.298$ and $\omega=2.5$ respectively for the case when there is no heating. Unless otherwise specified the values shown correspond to taking a timestep $d \tau=T_{\text {per }} / 256$ (with $\left.T_{\text {per }}=2 \pi / \omega_{0}\right), N=32, Y_{\max }=40$ and 2048 Fourier modes. The wall motion is described by $F_{a}(\tau, X)=h(X) q(\tau)$ and $q(\tau)=\sin \left(\omega_{0} \tau\right)$.

When $\omega=2$ the analysis predicts a stable wave, when $\omega=2.298$ the wave is neutrally stable and when $\omega=2.5$ an unstable wave growing downstream with $X$ is generated. The numerical solutions in figures $6-8$ are in accord with the predictions.

In figures 9-11 we present similar results but this time when there is no vibrator and only localised (unsteady) heating taking $F_{a}=0$ and $g(\tau, X)=h(X) q(\tau)$ and $q(\tau)=$ $\sin \left(\omega_{0} \tau\right)$. The analytical results predict stable, neutral, and unstable waves as before and as shown in the numerical results.

In figure 12 we present the temperature perturbation $\theta_{a}\left(\tau, X, Y=Y_{j}\right)$ for the unstable case of $\omega=2.5$ at various times and with no vibrator $\left(F_{a}=0\right)$ using the same localised heating function as in figure 11. Even though the frequency corresponds to the case of an unstable spatially (downstream) growing wave, the instability is confined to just the velocity and pressure field and not the temperature perturbation as predicted by the analysis. 

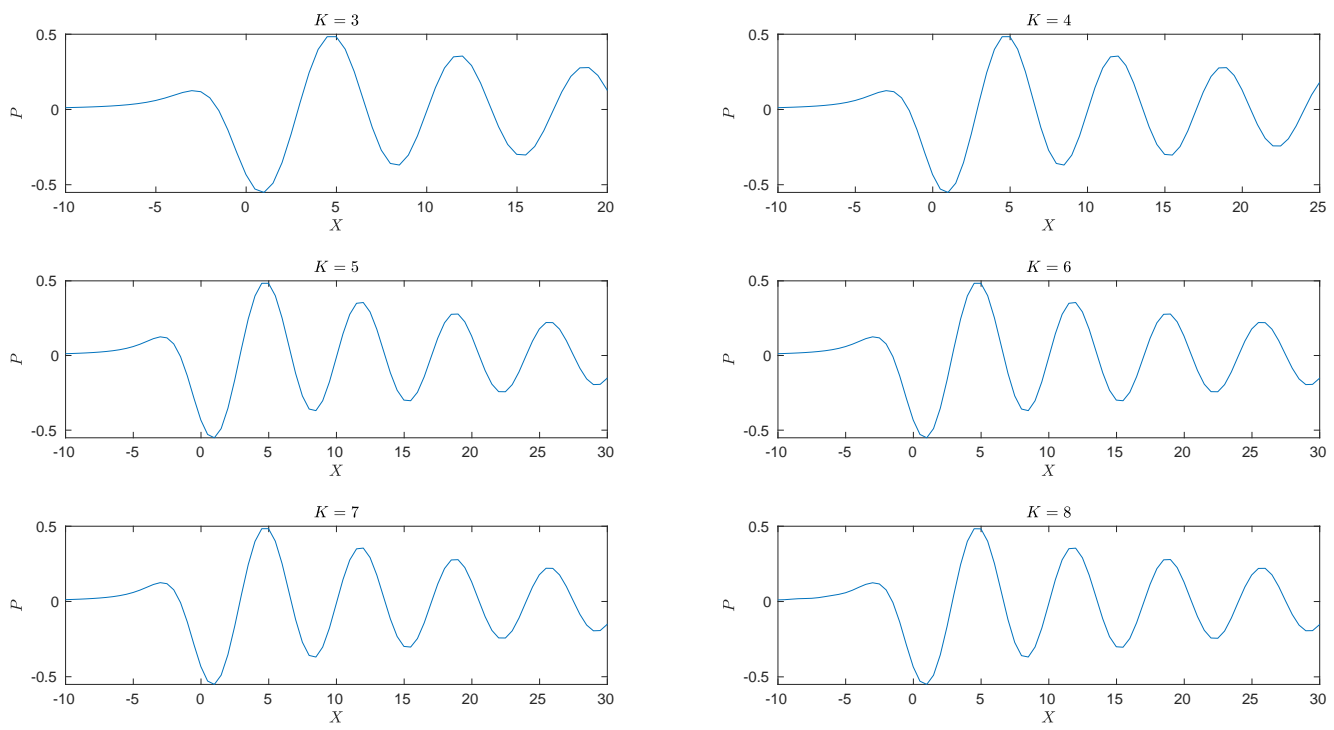

Figure 6: Results for the pressure perturbation with $\omega_{0}=2$ (stable) at times $\tau=(K+$ $\left.\frac{1}{4}\right) T_{p e r}, K=3,4,5,6,7,8$, with $g(\tau, X)=0$ (no heating).
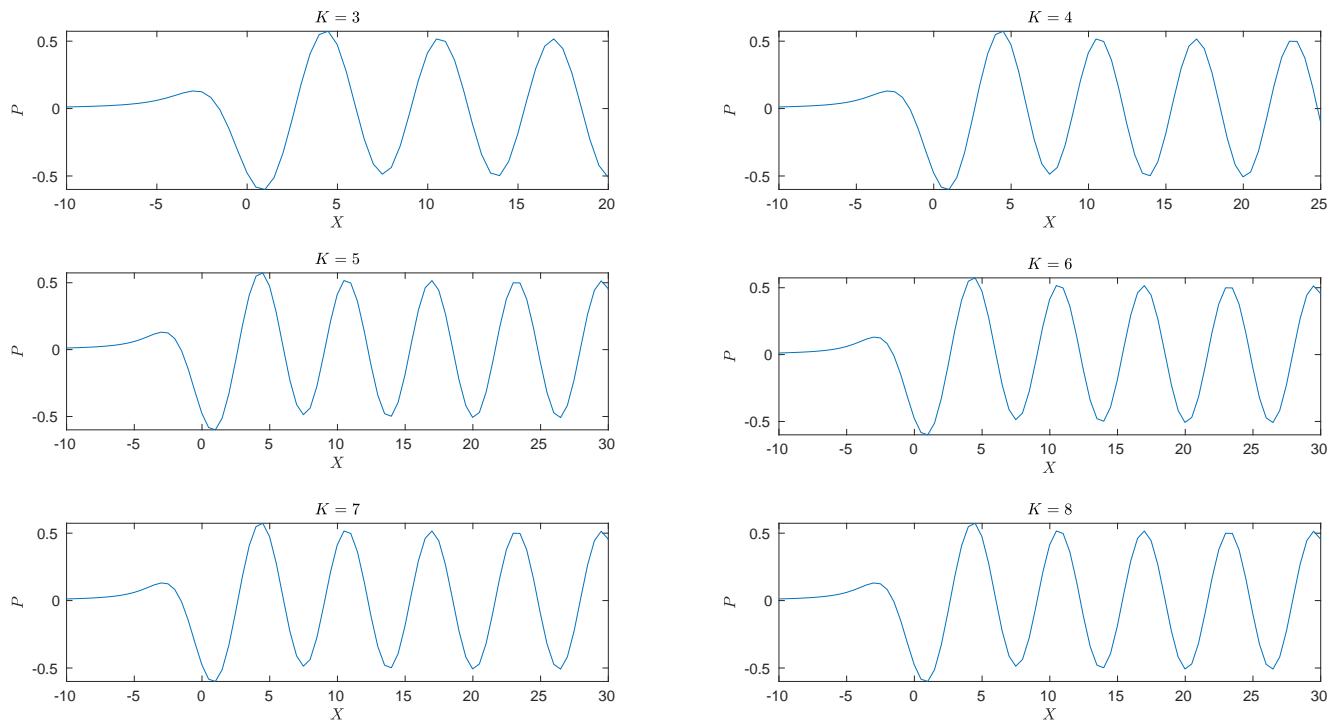

Figure 7: Results for the pressure perturbation with $\omega_{0}=2.298$ (neutral) at times $\tau=$ $\left(K+\frac{1}{4}\right) T_{p e r}, K=3,4,5,6,7,8$, with $g(\tau, X)=0$ (no heating).

\subsection{Tollmien-Schlichting wave cancellation}

In this section we present results showing Tollmien-Schlicting wave cancellation taking place with appropriately chosen localised temperature forcing. The analysis suggests that cancellation will take place provided that the wall forcing is chosen according to (2.31). We can formally invert this to find

$$
g_{T C}(\tau, X)=-\frac{1}{4 \pi^{2} i} \int_{-\infty}^{\infty} \int_{L} \frac{h^{\dagger}(k) \omega_{0} \operatorname{Ai}\left(\eta_{0}\right)(i k P r)^{1 / 3}}{\left(\omega^{2}+\omega_{0}^{2}\right) \int_{\eta_{0}}^{\infty} \operatorname{Ai}(\eta) d \eta} e^{\omega \tau+i k X} d \omega d k
$$



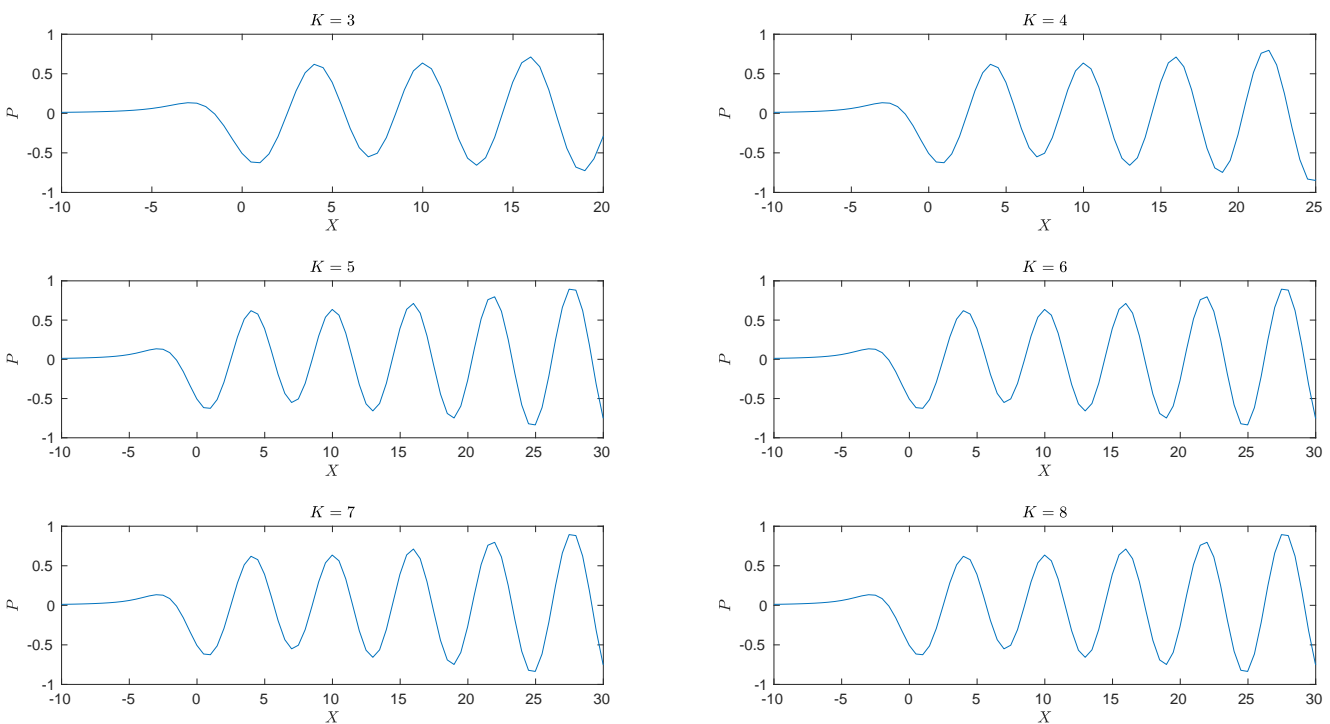

Figure 8: Results for the pressure perturbation with $\omega_{0}=2.5$ (unstable) at times $\tau=$ $\left(K+\frac{1}{4}\right) T_{p e r}, K=3,4,5,6,7,8$, with $g(\tau, X)=0$ (no heating).
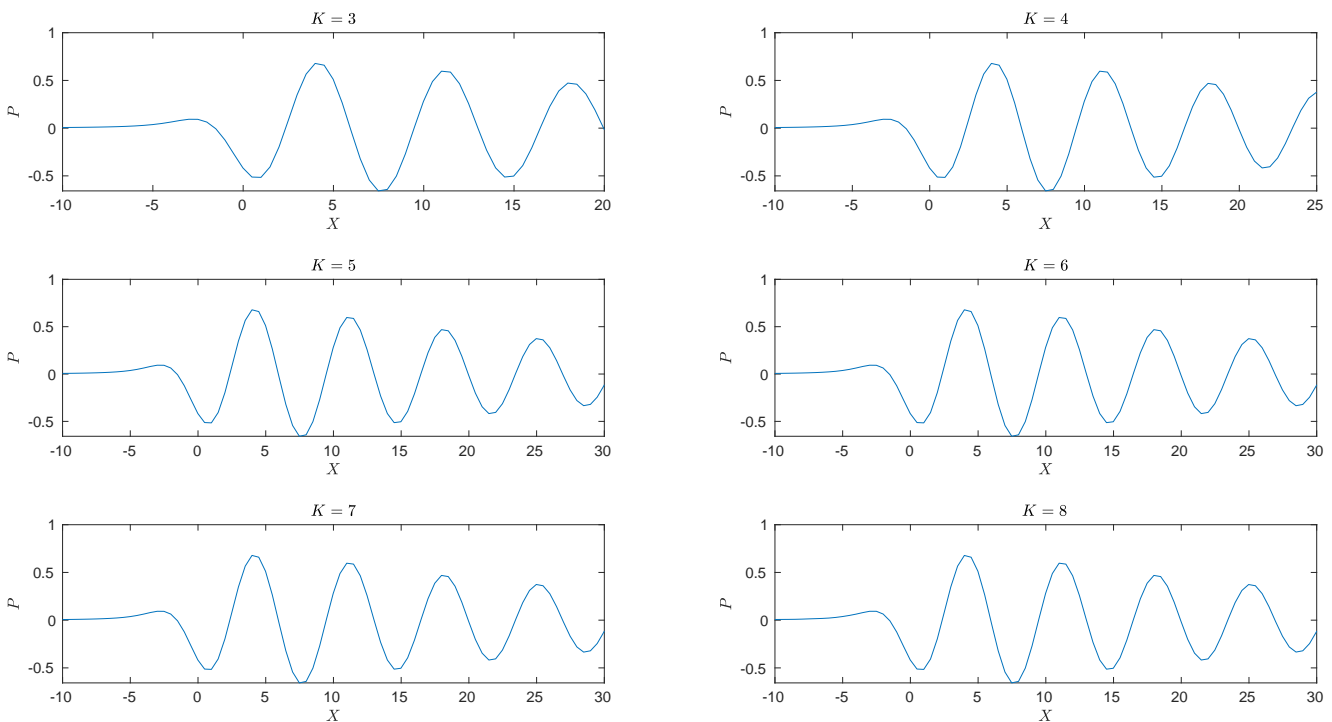

Figure 9: Results for the pressure perturbation with $\omega_{0}=2$ (stable) at times $\tau=(K+$ $\left.\frac{1}{4}\right) T_{p e r}, K=3,4,5,6,7,8$ and no vibrator, forced by unsteady heating.

where $L$ is a contour to the right of all singularities of the integrand in the complex $\omega$ plane. As far as the integral with respect to $\omega$ is concerned the integrand has poles at $\omega= \pm i \omega_{0}$ and when

$$
\int_{\eta_{0}}^{\infty} \operatorname{Ai}(\eta) d \eta=0
$$



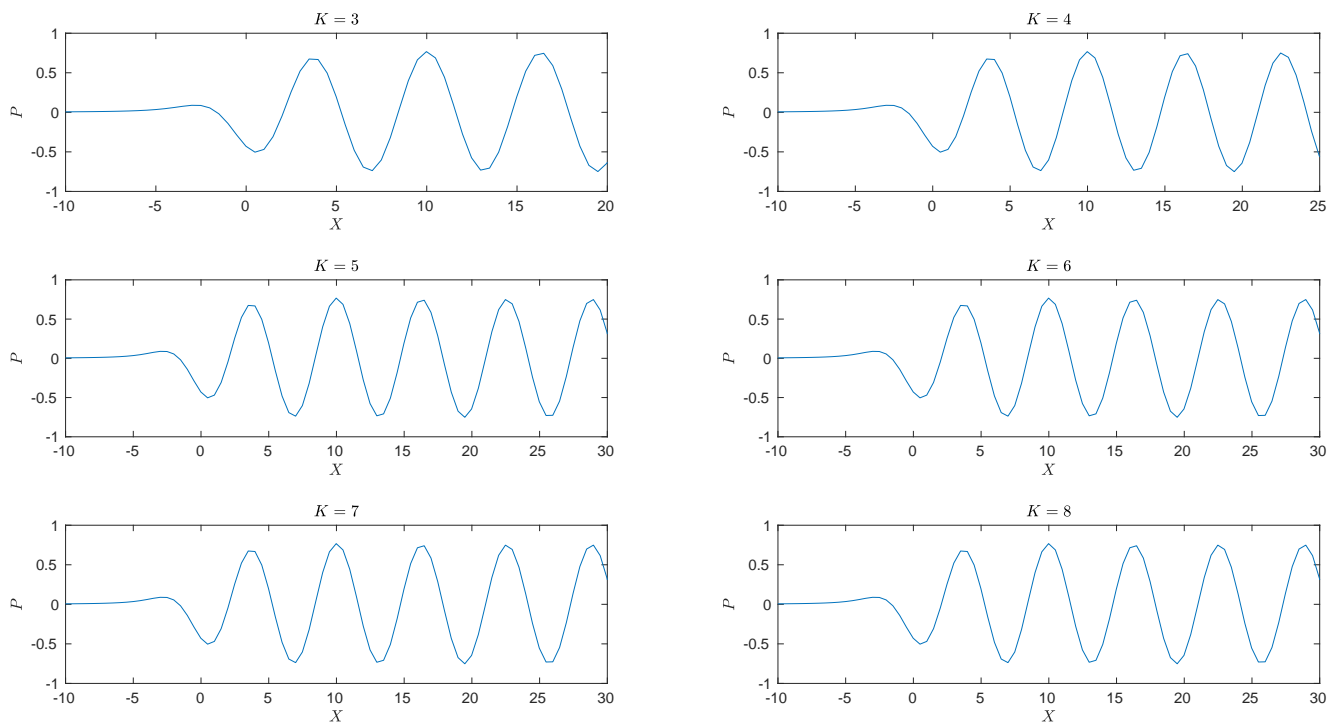

Figure 10: Results for the pressure perturbation with $\omega_{0}=2.298$ (neutral) at times $\tau=\left(K+\frac{1}{4}\right) T_{p e r}, K=3,4,5,6,7,8$ and no vibrator, forced by unsteady heating.
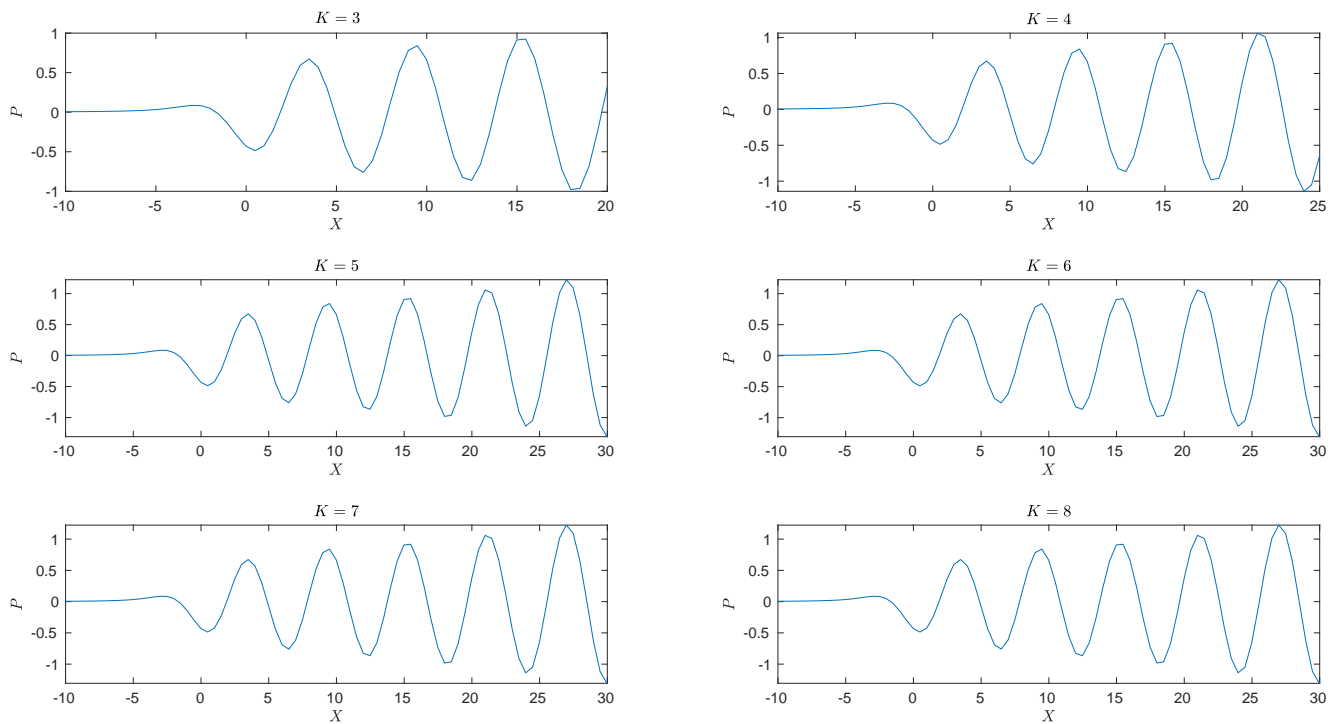

Figure 11: Results for the pressure perturbation with $\omega_{0}=2.5$ (unstable) at times $\tau=$ $\left(K+\frac{1}{4}\right) T_{p e r}, K=3,4,5,6,7,8$ and no vibrator, forced by unsteady heating.

The function $\int_{z}^{\infty} \operatorname{Ai}(\xi) d \xi$ is the Generalised Airy Function and it is known that the zeros of this function form an infinite countable set and arise as complex conjugate pairs with negative real values. The first few such values are tabulated in Table 1 . If we label these zeros as $b_{j}=\left|b_{j}\right| e^{-i \pi+i \psi_{j}}$ then from the definition $\eta_{0}$ we find that

$$
\omega=\omega_{6 j}=\frac{\left|b_{j}\right| e^{-\frac{2 \pi i}{3}+i \psi_{j}}(i k)^{2 / 3}}{\operatorname{Pr}^{1 / 3}} .
$$



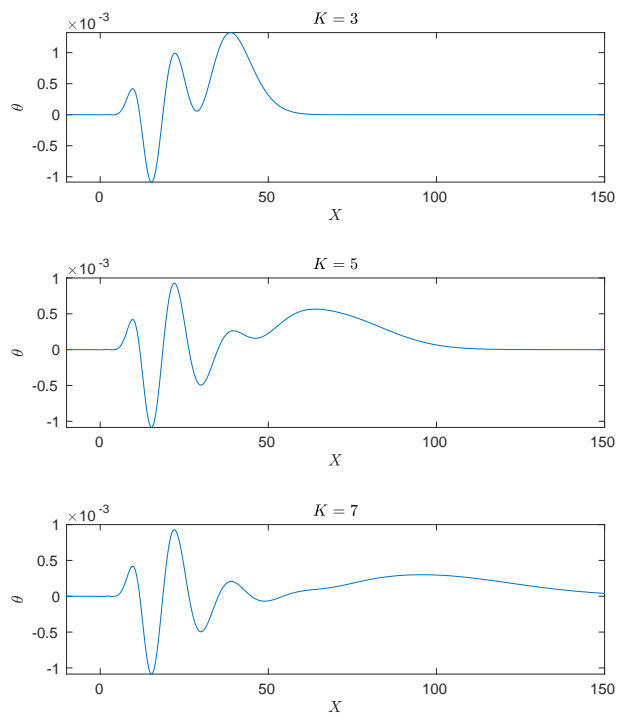
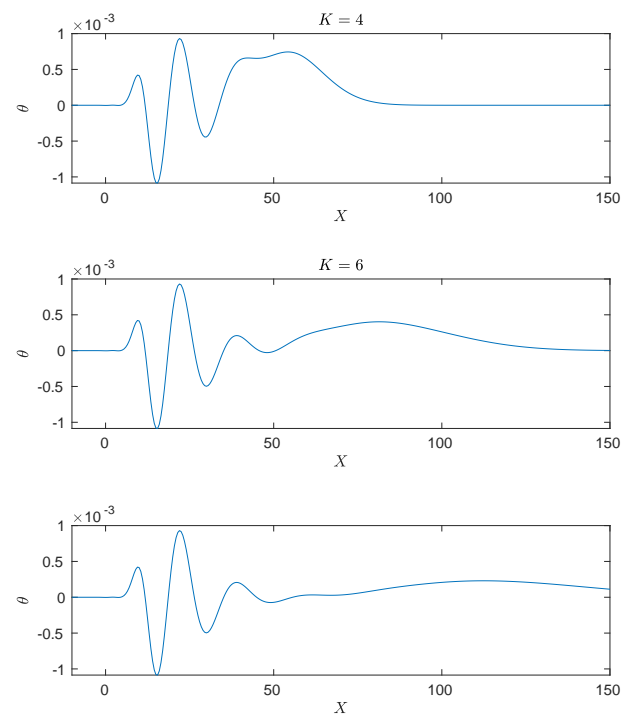

Figure 12: Temperature profile $\theta\left(X, Y_{j}, \tau\right)$ with $g(X, \tau)=h(X) q(\tau), h(x)=0$ and $\omega=2.5$ with $Y_{j} \approx 7.759$ at times $\tau=\left(K+\frac{1}{4}\right) T_{\text {per }}, K=3,4,5,6,7,8$.

Importantly we note from (4.2) that the real part of $\omega_{6 j}$ remains negative for $j=1,2, \ldots$

Hence inverting the inner integral in (4.1) gives

$$
\begin{aligned}
g_{T C}(\tau, X) & =-\frac{1}{2 \pi} \int_{-\infty}^{\infty} \frac{h^{\dagger}(k) \operatorname{Ai}\left(\eta\left(i \omega_{0}(k), k\right)\right)(i k P r)^{1 / 3}}{2 i \int_{\eta_{0}\left(i \omega_{0}, k\right)}^{\infty} \operatorname{Ai}(\eta) d \eta} e^{i \omega_{0} \tau+i k X} d k \\
& +\frac{1}{2 \pi} \int_{-\infty}^{\infty} \frac{h^{\dagger}(k) \operatorname{Ai}\left(\eta\left(-i \omega_{0}(k), k\right)\right)(i k P r)^{1 / 3}}{2 i \int_{\eta_{0}\left(-i \omega_{0}, k\right)}^{\infty} \operatorname{Ai}(\eta) d \eta} e^{-i \omega_{0} \tau+i k X} d k \\
& +\sum_{j=1}^{\infty} \int_{-\infty}^{\infty} \frac{h^{\dagger}(k)(i k) \omega_{0}}{\left(\omega_{0}^{2}+\omega_{6 j}^{2}\right) \operatorname{Pr}^{1 / 3}} e^{\omega_{6 j} \tau+i k X} d k
\end{aligned}
$$

However for the numerical computations instead of the exact cancellation function given by (4.3) an approximation $g_{T C N}(\tau, X)$ was used, which is obtained by ignoring the terms in the summation. In the Fourier transform space the function $g_{T C N}^{\dagger}(\tau, k)$ is given by

$g_{T C N}^{\dagger}(\tau, k)=h^{\dagger}(k) \frac{(i k P r)^{1 / 3} \operatorname{Ai}\left(\eta_{0}\left(-i \omega_{0}, k\right)\right)}{2 i \int_{\eta_{0}\left(-i \omega_{0}, k\right)}^{\infty} \operatorname{Ai}(\eta) d \eta} e^{-i \omega_{0} \tau}-h^{\dagger}(k) \frac{\operatorname{Ai}\left(\eta_{0}\left(i \omega_{0}, k\right)\right)(i k P r)^{1 / 3}}{2 i \int_{\eta_{0}\left(i \omega_{0}, k\right)}^{\infty} \operatorname{Ai}(\eta) d \eta} e^{i \omega_{0} \tau}$.

In figure 13 we show the result of wave cancellation in action. The wall motion is taken to be the same as that for figure 8 .

In figures 14 we have compared wave cancellation with and without localised heating. Figures 14(a) and 15(a) show the effect on the amplitude of the wave at two instants in time, by choosing $g(\tau, X)$ according to (4.4). In figures $14(\mathrm{~b})$ and $15(\mathrm{~b})$ the comparison is over an extended range of $X$. In the earlier figures only a restricted range of $X$ is shown and this corresponds to the developing Tollmien-Schlichting wave. Over the extended range, the full response including a faster growing convective wave packet is clearly visible. This arises from the transient part of the signal and is present for all the cases (stable, neutral and unstable) studied, and it can be seen that the amplitude of the wave packet is orders of magnitude larger than the Tollmien-Schlichting wave amplitude. 


$\begin{array}{cc}j & b_{j} \\ 1 & -4.10700-1.14416 i \\ 2 & -4.10700+1.14416 i \\ 3 & -6.79815-1.03516 i \\ 4 & -6.79815+1.03516 i \\ 5 & -9.03091-0.96941 i \\ 6 & -9.03091+0.96941 i\end{array}$

Table 1: First few zeros of $\int_{\xi}^{\infty} \operatorname{Ai}(t) d t$ denoted by $b_{j}$. Note that these arise as complex conjugate pairs with negative real parts.
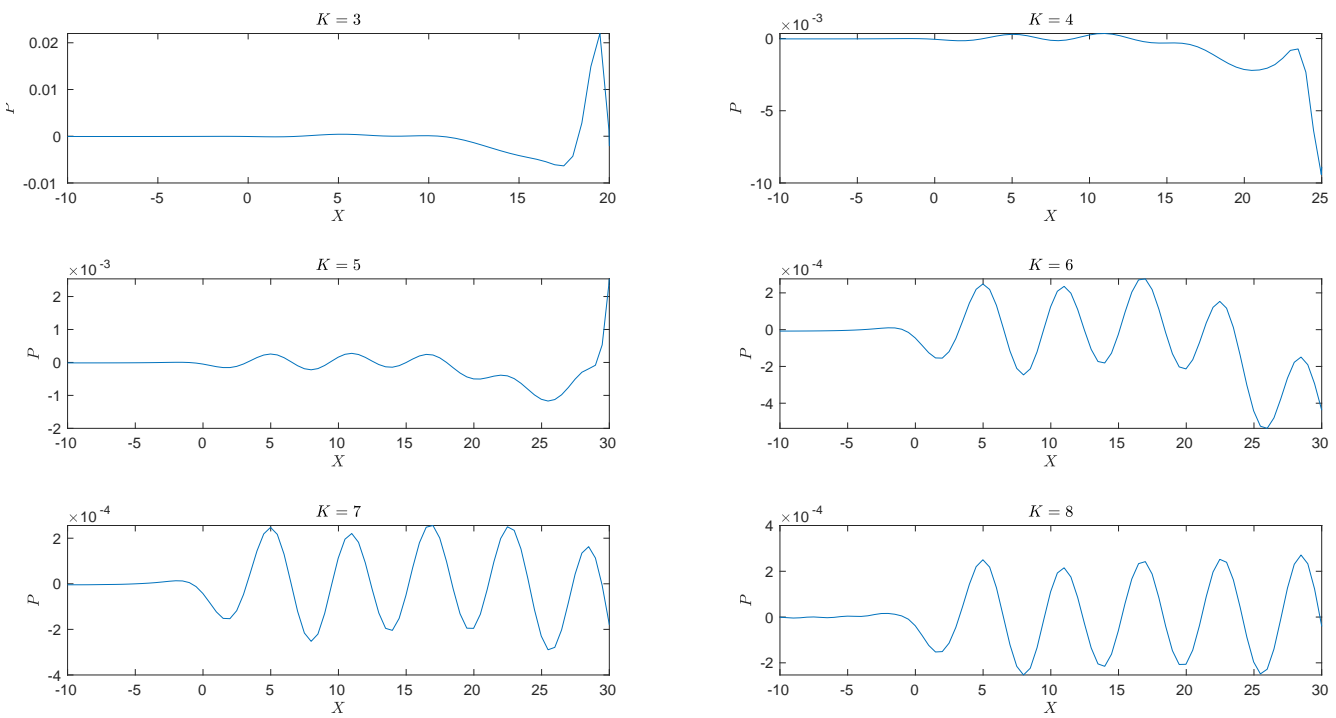

Figure 13: The pressure perturbation $P_{a}(\tau, X)$ with $\omega=2.5$ and T-S cancellation in action using the heating function given by (4.4) at times $\tau=\left(K+\frac{1}{4}\right) T_{p e r}, K=$ $3,4,5,6,7,8$.

With wave cancellation using (4.4), the amplitude of this wave packet is diminished but remains significant as figure $14(\mathrm{~b})$ shows.

In figure 16 the comparisons are also shown for the stable case with $\omega_{0}=2$ and in figure 17 for the neutral case with $\omega=2.298$.

The formula given by (4.3) predicts a complete cancellation of the signal, as seen in figures 15(a), 16(a) 17(a) for instance. However on using the approximate formula in (4.4) although the Tollmien-Schlichting wave amplitude is significantly reduced, as seen by a comparison of figures 13 and 8, the wave packet amplitude is still very large, as figures 15(b), 16(b), 17(b) show.

It is possible that taking more terms in the expansion may help in reducing the amplitude of the wave packet but limitations arise because of numerical precision and roundoff errors. This is also one of the reasons why we chose the wall function to be a smooth Gaussian hump. With a triangular hump as used by Terent'ev (1984) and given in (3.3), the computations were particularly sensitive to the growth of the high wavenumber components impacting on the wave packet amplitude. This can be seen in figure 18, which shows the Fourier transform amplitude as a function of the wavenumber at two times, 


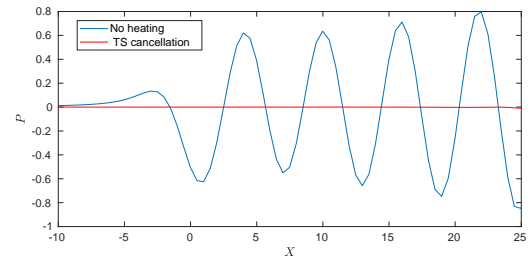

(a)

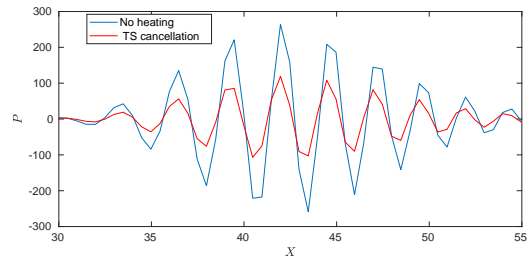

(b)

Figure 14: Comparisons of the pressure perturbation with $\omega=2.5$ at $T=(4+1 / 4) T_{\text {per }}$ with and without wave cancellation using (4.4): (a) for the restricted range in $x$, and in (b) over an extended range showing the wave packet.

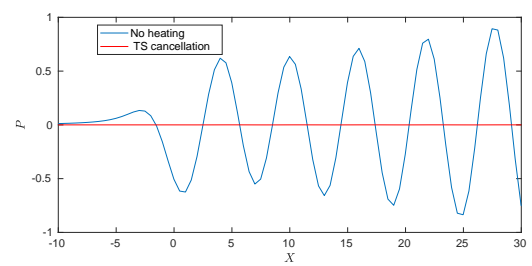

(a)

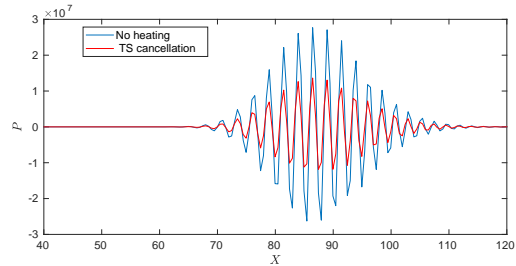

(b)

Figure 15: Comparison of the pressure perturbations with $\omega=2.5$ at $T=(8+1 / 4) T_{\text {per }}$ with and without wave cancellation using (4.4): (a) for restricted range in $x$, and in (b) over an extended range showing the wave packet.

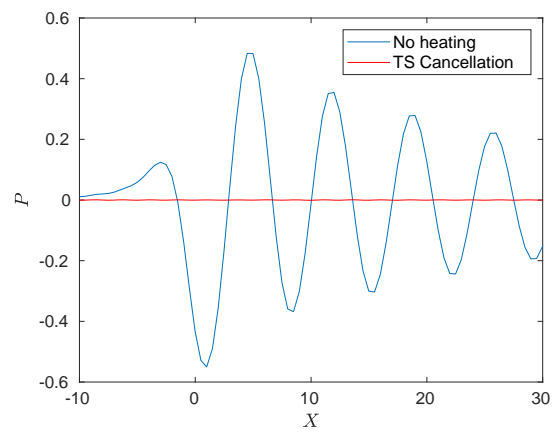

(a)

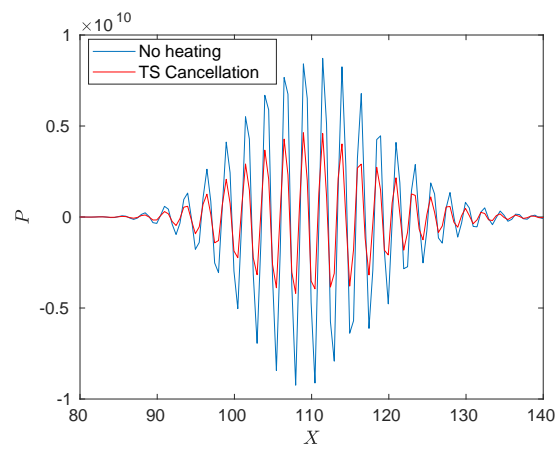

(b)

Figure 16: Comparison of the pressure perturbations at $T=(8+1 / 4) T_{\text {per }}$ with and without wave cancellation using (4.4) for the stable case with $\omega_{0}=2$. (a) for restricted range in $x$, and in (b) over an extended range showing the wave packet.

comparing the results for the Gaussian hump with the triangular hump (3.3). The figure 18 initially shows two peaks, one near $k=1$ corresponding to the Tollmien-Schlichting wave, and other near $k=2.5$ in accordance with figure 3. For a triangular hump, the growth of the high wavenumber components meant that computations and in particular the behaviour of the wave packet, could not be adequately resolved after a short time. We restrict attention in these results to $|k| \leqslant 2 \pi$; the inclusion of a larger wavenumber range would require even further decreases of the temporal step in the numerical calculations.

Extensive grid size and other checks were carried out as documented in the accompany- 


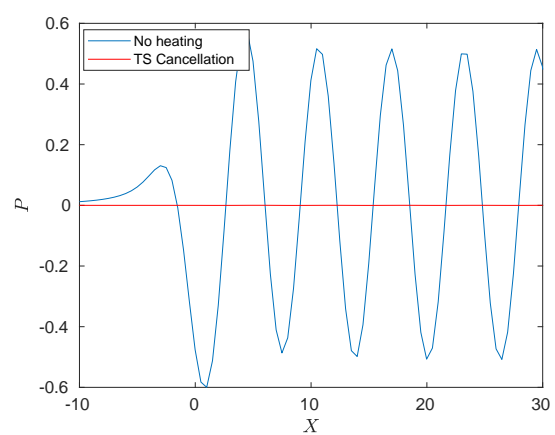

(a)

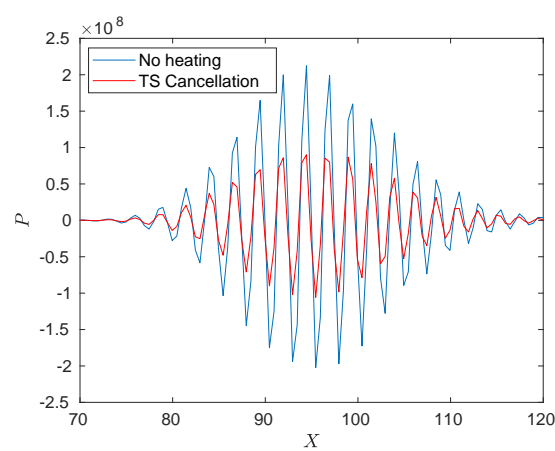

(b)

Figure 17: Comparison of the pressure perturbations at $T=(8+1 / 4) T_{p e r}$ with and without wave cancellation using (4.4) for the neutral case with $\omega_{0}=2.298$ (a) for restricted range in $X$, and in (b) over an extended range showing the wave packet.

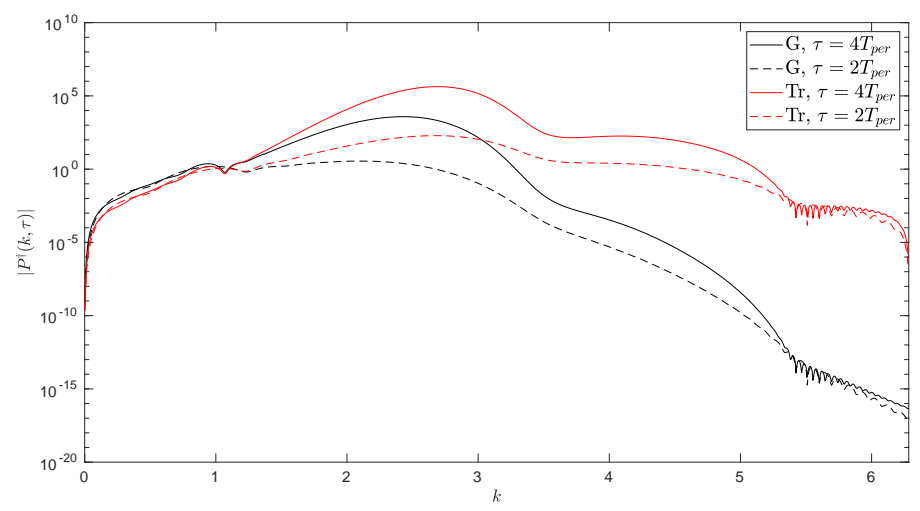

Figure 18: Plots of the function $\left|P^{\dagger}(\tau, k)\right|$ against $k$ at times $\tau=2 T_{\text {per }}$ (dotted line) and $\tau=4 T_{\text {per }}$ (solid line) for the Gaussian hump (labelled $G$ ) and triangular hump shape (labelled $T r$ ).

ing supplementary material. It is noted that the wave packet amplitude is most sensitive to changes in the time steps $d \tau$ and when using other integration schemes. Other parts of the signal including the Tollmien-Schlichting wave appear to be fully resolved when varying the grid parameters and timesteps.

\section{Conclusions}

The most significant finding in this study is that we have shown that it is possible to chose a localised temperature profile which is able to cancel out Tollmien-Schlichting waves. A modified version of the exact heating profile was shown to be effective in radically reducing the amplitude of the unstable Tollmien-Schlichting wave generated. Whilst the analysis performed is linear and thus restricted to small amplitudes, it would be interesting to try applying the idea for a full nonlinear simulation. In principle the same idea may be extended also for other situations including three-dimensional effects as well as Tollmien-Schlicting wave generation by other means. However, in a real flow 
Tollmien-Schlichting waves can be excited through multiple mechanisms and to be able to predict wave-cancellation profiles for a more general situation is a much more complex task. A number of problems for further study are suggested by the results presented here and in the first instance it would be interesting to revisit the experiments of Liepmann et al. (1982) and Liepmann \& Nosenchuck (1982) using Navier-Stokes simulations, and incorporating the analytic formula for wave cancellation wall heating functions given here.

We have also calculated the receptivity coefficients due to Tollmien-Schlicting waves generated by the effects of wall vibration as well as localised heating for a special case of sinusoidal temporal forcing as given by the expressions $(2.44 a),(2.44 b)$.

The original motivation for the current study was the desire to fully understand the wave packets which caused difficulties in simulations of the unsteady triple-deck equations for nonlinear mean flows as in the work of Logue et al. (2014) where also the wave packet was not fully resolved. By choosing to focus on a linear system where it is possible to proceed analytically as with the equations studied here, we have found that in order to resolve the wave packet in our linear system, extremely small timesteps are required. The timesteps used in Logue et al. (2014) are much larger in comparison. Also we found that the high frequency spatial components are amplified because of the numerical techniques used, and the growth of these also leads to difficulties in performing long time accurate computations.

Finally one other observation arising from the numerical results presented here is that the transient part of the signal leading to the formation of the downstream travelling wave packet has an order of magnitude larger amplitude as compared to the TollmienSchlichting wave amplitude. This is not too dissimilar to what is observed in the experimental studies by Gaster \& Grant (1975) where disturbances were introduced by impulsively injecting at some upstream location on the flat plate. This initiated a wave packet which propagated downstream. The frequency power spectrum in figure 2 of Gaster \& Grant (1975) shows two peaks as in our figure 18, and in the signal traces in figure 4 of Gaster \& Grant (1975), only the wave packet can be seen. The amplitude of the Tollmien-Schlichting wave excited is an order of magnitude smaller as their figure 2 also shows.

\section{Acknowledgements}

JSBG would like to thank Prof. P. Hall (Monash University), Prof. A. Ruban (Imperial College), Prof. F.T. Smith (UCL), and Dr Y. Bhumkar (IIT Bhubaneshwar) for helpful suggestions and discussions on the work and the results presented in this paper. JSBG is also grateful to the Sydney Mathematics Research Institute (SMRI) at the University of Sydney for hosting a visit in 2019 where part of this work was completed. GB acknowledges the support provided by the EPSRC Centre for Doctoral Training in Industrially Focused Mathematical Modelling (EP/L015803/1).We would like to thank the referees for their useful and helpful comments which helped to improve the manuscript. One of the referees kindly directed us to the work of van Dyke (1952) and the relevant page in Neiland et al. (2007) which helped us in improving the appendix when discussing the combined Prandtl, Dorodnitsyn-Howarth transformation.

Declaration of Interests The authors report no conflict of interest. 


\section{References}

\section{REFERENCES}

Aljohani, A. F. \& GajJar, J. S. B. $2017 a$ Subsonic flow past localised heating elements in boundary layers. J. Fluid Mech. 821, R2.

Aljohani, A. F. \& GajJar, J. S. B. $2017 b$ Subsonic flow past three-dimensional localised heating elements in boundary layers. Fluid Dyn. 49(6), 065503.

Aljohani, A. F. \& GajJar, J. S. B. 2018 Transonic flow over localised heating elements in boundary layers. J. Fluid Mech. 844, 746-765.

Cassel, K. W., Ruban, A. I. \& Walker, J. D. A. 1995 An instability in supersonic boundarylayer flow over a compression ramp. J. Fluid Mech. 300, 265-285.

De Tullio, N. \& Ruban, A. I. 2015 A numerical evaluation of the asymptotic theory for subsonic compressible boundary layers. J. Fluid Mech. 771, 520-546.

Fletcher, A. J. P., Ruban, A. I. \& Walker, J. D. A. 2004 Instabilities in supersonic compression ramp flow. J. Fluid Mech. 517, 309-330.

GajJAR, J. S. B. 1996 Nonlinear stability of non-stationary cross-flow vortices in compressible boundary layers. Stud. Appl. Maths. 96, 53-84.

GASTER, M. 1965 On the generation of spatially growing instability waves in the boundary layer. J. Fluid Mech. 22, 433-441.

Gaster, M. \& Grant, I. 1975 An experimental investigation of the formation and development of a wave packet in a laminar boundary layer. Proc. Roy. Soc. A 347, 253-269.

Goldstein, M. E. 1983 The evolution of Tollmien-Sclichting waves near a leading edge. J. Fluid Mech. 127, 59-81.

Goldstein, M. E. 1985 Scattering of acoustic waves into Tollmien-Schlichting waves by small streamwise variations in surface geometry. J. Fluid Mech. 154, 509-529.

Koroteev, M. V. \& Lipatov, I. I. 2009 Supersonic boundary layer in regions with small temperature perturbations on the wall. SIAM J. Appl. Maths 70, 1139-1156.

Koroteev, M. V. \& Lipatov, I. I. 2012 Local temperature perturbations of the boundary layer in the regime of free viscous inviscid interaction. J. Fluid Mech. 707, 595-605.

Koroteev, M. V. \& Lipatov, I. I. 2013 Steady subsonic boundary layer in domains of local surface heating. App. Math. Mech. 77, 486-493.

Liepmann, H., Brown, G. \& Nosenchuck, D. 1982 Control of laminar instability waves using a new technique. J. Fluid Mech. 118, 187-200.

Liepmann, H. \& Nosenchuck, D. 1982 Active control of laminar turbulent transition. J. Fluid Mech. 118, 201-204.

LIN, C. C. 1955 The theory of hydrodynamic stability. Cambridge University Press.

LipATOV, I. I. 2006 Disturbed boundary layer flow with local time-dependent surface heating. Fluid Dyn. 41, 55-65.

LÖFdAHL, L. \& GAD-EL-HAK, M. 1999 MEMS applications in turbulence and flow control. Progress in Aerospace Sciences 35 (2), 101-203.

Logue, R. P. 2008 Numerical studies of bifurcation in flows governed by the triple-deck and other equations. PhD thesis, University of Manchester.

Logue, R. P., GajJar, J. S. B. \& Ruban, A. I. 2014 Instability of supersonic compression ramp flow. Philos. Trans. R. Soc. London Ser. A 372 (2020), 20130342.

Messiter, A. F. 1970 Boundary-layer flow near the trailing edge of a flat plate. SIAM J. Appl. Math. 18, 241-257.

NeIland, V. Y. 1969 Theory of laminar boundary layer separation in supersonic flow. Izv. Akad. Nauk SSSR, Mech. Zhidk. Gaza 4, 53-57.

Neiland, V. Y., Bogolepov, V., Dudin, G. \& Lipatov, I. 2007 Asymptotic theory of supersonic viscous gas flows. Elsevier, Butterworth-Heinemann.

PrandTL, L. 1904 Über flüssigkeitsbewegung bei sehr kleiner reibung. In Verh. III. Intern. Math. Kongr., Heidelberg, Teubner, Leipzig, 1905, pp. 484-491.

RubAn, A. I. 1984 On Tollmien-Schlichting wave generation by sound. Izv. Akad. Nauk SSSR Mekh. Zhidk. Gaza 5, 44-52. 
Ruban, A. I., Bernots, T. \& Kravtsova, M. A. 2016 Linear and nonlinear receptivity of the boundary layer in transonic flows. J. Fluid Mech. 786, 154-189.

Schubauer, G. B. \& Skramstad, H. K. 1948 Laminar boundary layer oscillations and transition on a flat plate. NACA Rep. 909.

Seddougui, S., Bowles, R. \& Smith, F. 1991 Surface-cooling effects on boundary-layer instability and upstream influence. European Jour. of Fluids/B 10, 117-145.

Smith, F. T. 1979 On the non-parallel flow stability of the Blasius boundary layer. Proc. Roy. Soc. London Ser. A 366, 91-109.

Smith, F. T. 1982 On the high Reynolds number theory of laminar flows. IMA Journal of Applied Mathematics 28 (3), 207-281.

Stewartson, K. 1974 Multistructured boundary layers on flat plates and related bodies. Advances in Applied Mechanics 14, 145 - 239.

Stewartson, K. 1981 D'Alembert's Paradox. SIAM Rev. 23 (2), 308-343.

Stewartson, K. \& Williams, P. 1969 Self-induced separation. Proc. Roy. Soc. A 312, 181206.

Terent'Ev, E. D. 1981 The linear problem of a vibrator in a subsonic boundary layer. Prikl. Mat. i Mekh. 45, 1049-1055.

TERENT'Ev, E. D. 1984 Linear problem of a vibrator peforming harmonic oscillations at supercritical frequencies in a subsonic boundary layer. Prikl. Mat. i Mekh. 48, 264-272.

Treviño, C. \& LiñÁn, A. 1996 The effects of displacement induced by thermal perturbations on the structure and stability of boundary-layer flows. Theoret. Comput. Fluid Dyn. 8, $57-72$.

Tutty, O. R. \& Cowley, S. J. 1986 On the stability and the numerical solution of the unsteady interactive boundary-layer equation. J. Fluid Mech. 168, 431-456.

VAN DyKe, M. 1952 Impulsive motion of an infinite plate in a viscous compressible fluid. $Z$. angew. Math. Phys. 3 (5), 343-353.

Walker, J. D. A., Fletcher, A. \& Ruban, A. I. 2006 Instabilities of a flexible surface in supersonic flow. Q. J. Mech. Appl. Math. 59 (2), 253-276.

\section{Appendix A. Combined Prandtl Dorodnitsyn-Howarth transform.}

Following van Dyke (1952), Neiland et al. (2007) we introduce the change of variables $\left(t_{*}, x_{*}, y_{3}\right) \rightarrow\left(T_{*}, X_{*}, y_{*}\right)$ where

$$
T_{*}=t_{*}, \quad X_{*}=x_{*}, \quad y_{*}\left(t_{*}, x_{*}, y_{3}\right)=\int_{f\left(t_{*}, x_{*}\right)}^{y_{3}} \rho_{*}\left(t_{*}, x_{*}, y\right) d y,
$$

and

$$
\rho_{*} v_{3}=v_{*}-\frac{\partial y_{*}}{\partial t_{*}}-u_{*} \frac{\partial y_{*}}{\partial x_{*}} .
$$

Note that we have introduced the independent variables $T_{*}=t_{*}, X_{*}=x_{*}$ for extra clarity in the discussion below, although in the main text we have retained $t_{*}$ and $x_{*}$ only.

The combined Prandtl Dorodnitsyn-Howarth given by (A 1),(A 2) and as used in the text, is a generalisation of the more familiar Dorodnitsyn-Howarth transform, see van Dyke (1952), Neiland et al. (2007), in which the lower limit in the integral for $y_{*}$ is zero. Details of how this transform reduces the continuity equation to one without $\rho_{*}$, and how the boundary conditions at the wall $y_{3}=f$ are satisfied, are given below.

Using the transformation we have

$$
\begin{aligned}
\frac{\partial}{\partial t_{*}} & =\frac{\partial}{\partial T_{*}}+\frac{\partial y_{*}}{\partial t_{*}} \frac{\partial}{\partial y_{*}}, \\
\frac{\partial}{\partial x_{*}} & =\frac{\partial}{\partial X_{*}}+\frac{\partial y_{*}}{\partial x_{*}} \frac{\partial}{\partial y_{*}},
\end{aligned}
$$




$$
\frac{\partial}{\partial y_{3}}=\rho_{*} \frac{\partial}{\partial y_{*}}
$$

Next integrating the continuity equation (2.3a) and making use of the boundary conditions

$$
u_{*}=0, \quad v_{3}=\frac{\partial f}{\partial t_{*}}, \quad \text { on } \quad y_{3}=f\left(t_{*}, x_{*}\right),
$$

we obtain

$$
\rho_{*} v_{3}=-\int_{f}^{y_{3}} \frac{\partial \rho_{*}}{\partial t_{*}} d y_{3}-\int_{f}^{y_{3}} \frac{\partial}{\partial x_{*}}\left(\rho_{*} u_{*}\right) d y_{3}+\rho\left(t_{*}, x_{*}, f\right) \frac{\partial f}{\partial t_{*}}\left(t_{*}, x_{*}\right) .
$$

Next we make use of the Leibniz's rule for differentiation under the integral sign in (A 5) together with the boundary conditions in (A 4) to give

$$
\begin{aligned}
\rho_{*} v_{3} & =-\left[\frac{\partial}{\partial t_{*}}\left(\int_{f}^{y_{3}} \rho_{*} d y_{3}\right)+\rho_{*}\left(t_{*}, x_{*}, f\right) \frac{\partial f}{\partial t_{*}}\left(t_{*}, x_{*}\right)\right] \\
& -\left[\frac{\partial}{\partial x_{*}}\left(\int_{f}^{y_{3}} \rho_{*} u_{*} d y_{3}\right)+\rho_{*}\left(t_{*}, x_{*}, f\right) u_{*}\left(t_{*}, x_{*}, f\right) \frac{\partial f}{\partial x_{*}}\left(t_{*}, x_{*}\right)\right] \\
& +\rho\left(t_{*}, x_{*}, f\right) \frac{\partial f}{\partial t_{*}}\left(t_{*}, x_{*}\right) \\
& =-\frac{\partial y_{*}}{\partial t_{*}}-\frac{\partial}{\partial x_{*}}\left(\int_{f}^{y_{3}} \rho_{*} u_{*} d y_{3}\right) .
\end{aligned}
$$

We now introduce

$$
u_{*}=\frac{\partial \Psi}{\partial y_{*}}
$$

and making use of the definition of $y_{*}$, from (A 6) we have

$$
\begin{aligned}
\rho_{*} v_{3} & =-\frac{\partial y_{*}}{\partial t_{*}}-\frac{\partial}{\partial x_{*}}\left(\int_{0}^{y_{*}} u_{*} d y_{*}\right) \\
& =-\frac{\partial y_{*}}{\partial t_{*}}-\frac{\partial \Psi}{\partial x_{*}}, \\
& =-\frac{\partial y_{*}}{\partial t_{*}}-\frac{\partial \Psi}{\partial X_{*}}-u_{*} \frac{\partial y_{*}}{\partial x_{*}} .
\end{aligned}
$$

Combining (A 2) with the last result we find that

$$
v_{*}=-\frac{\partial \Psi}{\partial X_{*}} .
$$

This now shows that the transformed continuity equation

$$
\frac{\partial u_{*}}{\partial X_{*}}+\frac{\partial v_{*}}{\partial y_{*}}=0
$$

is satisfied. The definition of $v_{*}$ in (A 2) also implies that $v_{*}=0$ on $y_{3}=f$ or $y_{*}=0$. Next the momentum equation (2.3b) together with the transformations (A 3) yields

$$
\begin{array}{r}
\rho_{*}\left(\frac{\partial u_{*}}{\partial T_{*}}+\frac{\partial y_{*}}{\partial t_{*}} \frac{\partial u_{*}}{\partial y_{*}}+u_{*}\left(\frac{\partial u_{*}}{\partial X_{*}}+\frac{\partial y_{*}}{\partial x_{*}} \frac{\partial u_{*}}{\partial y_{*}}\right)+\rho_{*} v_{3} \frac{\partial u_{*}}{\partial y_{*}}\right) \\
=-\frac{\partial p_{*}}{\partial X_{*}}-\frac{\partial y_{*}}{\partial x_{*}} \frac{\partial p_{*}}{\partial y_{*}}+\rho_{*} \frac{\partial}{\partial y_{*}}\left(\mu_{*} \rho_{*} \frac{\partial u_{*}}{\partial y_{*}}\right)
\end{array}
$$

which after using (A 2),(A $3 c),(2.3 c),(2.3 e)$ noting that $\partial p_{*} / \partial y_{*}=0$, reduces to $(2.6 b)$. The equation $(2.3 d)$ for $T_{*}$ is handled similarly. 